Revue des patrimoines

\title{
Les principes de la restauration d'instruments scientifiques : le cas du cercle méridien Gautier de l'observatoire de Rio de Janeiro
}

Marcus Granato et Françoise Le Guet Tully

\section{OpenEdition}

Journals

Édition électronique

URL : http://journals.openedition.org/insitu/3865

DOI : 10.4000/insitu.3865

ISSN : 1630-7305

Éditeur

Ministère de la Culture

Référence électronique

Marcus Granato et Françoise Le Guet Tully, « Les principes de la restauration d'instruments

scientifiques : le cas du cercle méridien Gautier de l'observatoire de Rio de Janeiro », In Situ [En ligne], 10 | 2009, mis en ligne le 19 mai 2009, consulté le 14 novembre 2019. URL : http:// journals.openedition.org/insitu/3865 ; DOI : 10.4000/insitu.3865

Ce document a été généré automatiquement le 14 novembre 2019.

\section{(c) $(1)$}

In Situ Revues des patrimoines est mis à disposition selon les termes de la licence Creative Commons Attribution - Pas d'Utilisation Commerciale - Pas de Modification 4.0 International. 


\title{
Les principes de la restauration d'instruments scientifiques : le cas du cercle méridien Gautier de l'observatoire de Rio de Janeiro
}

\author{
Marcus Granato et Françoise Le Guet Tully
}

\section{Réflexions générales à propos de la restauration d'instruments scientifiques}

\section{Questions de principes}

1 Selon le Comité pour la Conservation de l'ICOM ${ }^{1}$, la restauration est une intervention physique effectuée sur un objet en vue d'augmenter la durée de vie de ce dernier tout en respectant son intégrité matérielle, esthétique et fonctionnelle. Un objet restauré correctement se rapprocherait donc autant que faire se peut de l'état initial dans lequel il était au moment de sa conception et de sa fabrication. Selon l'ICOM, grâce aux techniques d'intervention qu'il met en œuvre, le conservateur sauvegarde et redonne leur fonctionnalité à des objets qui pourront dès lors être montrés au public dans le cadre soit d'une thématique spécifique soit d'une période historique particulière.

2 Mais les instruments scientifiques entrent-ils dans ce cadre général ? Pour Miniati² ${ }^{2}$ la restauration d'instruments scientifiques aurait de nombreuses similitudes avec celle des œuvres d'art dans la mesure où de nombreux instruments, et notamment les plus anciens, présentent des caractéristiques esthétiques, décoratives et matérielles analogues à celles des œuvres d'art. Par contre dans certains cas, comme par exemple lorsque l'instrument à restaurer doit être remis en état de marche, les procédures à mettre en œuvre seraient totalement différentes.

3 Selon Sebastian ${ }^{3}$, les interventions sur des instruments scientifiques historiques ne devraient être effectuées que lorsqu'elles sont strictement nécessaires à la sauvegarde 
et à la future conservation de l'objet. Sebastian reconnaît qu'il n'est pas toujours facile de faire comprendre à des non spécialistes de muséologie scientifique pourquoi il est indispensable de conserver l'intégrité d'une pompe hydraulique ou d'un microscope, pourquoi seuls le respect de cette intégrité et la compréhension de l'objet peuvent justifier l'ajout d'éléments neufs et pourquoi dans ce dernier cas il ne suffit pas de conserver la trace écrite de la modification dans le dossier documentaire de l'objet, mais que ce dernier doit en porter une trace physique pérenne. À titre de comparaison, Sebastian souligne qu'il n'est pas facile non plus pour un mécanicien qui intervient sur des équipements industriels d'apprendre à traiter avec la considération nécessaire des objets culturels patrimoniaux.

4 Au-delà de ces questions de principe, il est indispensable préalablement à la restauration d'un instrument scientifique d'entreprendre une étude documentaire approfondie sur l'objet en question. Il convient en effet de collecter un maximum d'informations sur le(s) principe(s) physique(s) selon le(s)quel(s) il a été conçu, sur sa ou ses fonction(s), son ou ses constructeur(s), sa datation, ses usages, les transformations qu'il a subies au cours du temps, etc. Ceci nécessite des recherches à la fois dans la littérature spécialisée et dans les divers fonds d'archives qui concernent l'instrument et, le cas échéant, des entretiens avec d'anciens utilisateurs ou des témoins. Toutes les décisions relatives à la restauration de l'objet s'appuieront en effet sur un dossier documentaire qui devra indiquer aussi précisément que possible fonction, principe, constructeur et histoire de l'objet à restaurer.

5 Une fois prise la décision de restaurer l'instrument, le conservateur et le restaurateur doivent s'efforcer de définir ensemble la méthode de restauration la plus appropriée, particulièrement en cas de remplacement de parties manquantes ou de réparation de parties endommagées. La consultation de périodiques et de catalogues de l'époque de fabrication de l'instrument ainsi que de publications spécialisées dans les questions de restauration constitue un atout considérable pour mener à bien cette étape cruciale de la procédure.

6 Selon André ${ }^{4}$, les lignes directrices d'une éthique de restauration des instruments scientifiques devraient être les suivantes :

- collecter la documentation la plus large possible avant de commencer toute intervention sur l'objet ;

- avoir toujours en tête le principe d'intervention minimale ;

- respecter l'intégrité de l'objet, notamment en conservant autant d'éléments originaux que possible et en évitant d'ajouter des éléments neufs ;

- s'assurer que l'intervention est réversible.

7 S'il n'est pas toujours possible de suivre ces principes, l'important est cependant de comprendre l'objet et son fonctionnement et, le cas échéant, d'intégrer dans la réflexion la façon dont une fois restauré il sera présenté au public.

Le principe de réversibilité soulève de nombreuses questions, dont précisément celle de la définition d'une intervention réversible. Par ailleurs, si de nombreuses interventions opérées sur du patrimoine industriel ou scientifique sont de fait irréversibles - soudure, sablage, substitution d'un métal par un autre - ce n'est pas pour autant qu'elles doivent être totalement proscrites. Meehan ${ }^{5}$ discute longuement ce point dans le contexte spécifique du patrimoine industriel. Selon cet auteur, le démontage d'une machine constituerait un acte réversible générant un flot de connaissances sur l'objet lui-même - opération impossible à effectuer sur d'autres types de patrimoine. Meehan montre 
cependant au travers de nombreux exemples que souvent les procédures réversibles sont soit irréalisables, soit inappropriées. Selon lui les décisions concernant l'action à mener sur l'instrument devraient être guidées par un principe d'intervention minimale qui permette la préservation de ses composants originaux à long terme.

\section{Écoles de pensée en matière de restauration d'instruments scientifiques}

9 Tout comme celles d'autres domaines culturels, les pratiques de restauration des instruments scientifiques relèvent d'écoles de pensée distinctes dont les perspectives diffèrent quelque peu. L'une d'elles, mise en œuvre par les experts et chercheurs qui s'intéressent aux aspects historiques de la science, considère que l'objectif principal de la restauration est de retrouver la fonctionnalité de l'instrument. En d'autres termes, l'idée directrice est de remettre l'instrument dans son état de marche d'origine, cette fonctionnalité étant précisément ce qui le différencie des autres objets muséaux. Cette approche peut entraîner des altérations importantes de certains traits caractéristiques de l'objet. Une autre école de pensée, développée par les historiens des sciences et les restaurateurs d'art, préconise essentiellement de préserver les traces historiques inscrites dans l'objet, traces qui le plus souvent empêchent précisément l'objet de fonctionner.

10 C'est sans doute $\mathrm{Mann}^{6}$, du Science Museum de Londres, qui, dans la littérature que nous avons pu consulter, défend le mieux cette approche. Mann suppose que l'éthique qui gouverne la plupart des musées (d'art, d'archéologie, d'ethnographie, d'histoire, etc.) passe par la préservation des traces historiques et conclut que cette mission n'est pas celle des musées de science. Selon Mann une éthique nouvelle serait plus adaptée, éthique qui d'ailleurs serait mise en pratique par ses partisans, mais non énoncée explicitement. Celle-ci consisterait à privilégier l'exploitation de l'objet au bénéfice du public, y compris au détriment de la préservation des traces historiques qui y sont matériellement inscrites. Cette mutation de l'objectif premier s'appuierait sur un changement de concept de l'artefact qui, de porteur d'une trace matérielle, deviendrait porteur d'une trace fonctionnelle.

11 Toujours selon Mann, les objectifs poursuivis par les musées de science et de technologie permettraient d'accepter que soit détruit l'un des aspects de la mémoire portée par l'objet afin qu'un autre, plus important, puisse être révélé au public - au moyen par exemple d'un écorché de l'instrument ou de la machine ou de sa mise en marche. De ce point de vue, ces musées différeraient des autres dans la mesure où leur objectif premier serait d'expliquer le fonctionnement d'un objet plutôt que d'en assurer la conservation. Selon lui, dans les autres musées, même si certains objets possèdent un volet fonctionnel, ce n'est pas pour cette raison qu'ils sont intégrés dans une collection, mais pour leur valeur esthétique ou leur intérêt historique.

Selon cette école, les pratiques mises en œuvre au Science Museum de Londres et dans nombre d'autres musées des sciences et des techniques - avec des machines et des instruments présentés en écorché ou en fonctionnement - deviendraient des pratiques courantes destinées à développer la compréhension du public pour les objets exposés. Cependant l'auteur néglige le fait que les objets scientifiques y sont aussi conservés pour leur intérêt historique car ils permettent de mettre en évidence l'évolution des sciences et des techniques et contribuent à la réflexion sur cette évolution. 
13 À côté de cette tendance, il existe aussi au Royaume-Uni un point de vue plus conservateur, inspiré de la tradition de Ruskin \& Morris. Ainsi Newey ${ }^{7}$ préconise-t-elle l'utilisation de répliques qui selon elle seraient mieux à même de transmettre au public l'information relative à un objet que l'objet historique original en état de marche.

Comme le souligne Brenni $^{8}$, la restauration d'instruments scientifiques est une pratique récente qui manque encore d'une tradition bien établie. Plus souvent qu'il ne le faudrait, cette pratique s'intéresserait plus aux enjeux techniques de la restauration qu'à la valeur historique de l'objet à restaurer. Brenni constate que la littérature internationale sur ce sujet est réduite et que les quelques traités existants contribuent seulement à apporter une aide à la réparation des instruments. D'un côté les collectionneurs, les experts et les physiciens privilégient une restauration en profondeur des objets afin de retrouver en premier lieu leur fonctionnalité. De l'autre les restaurateurs d'art ont tendance à préconiser des restaurations très superficielles qui ne remplacent aucun élément et ne réparent pas l'objet. Pour Brenni ces deux approches sont trop radicales car elles aboutissent à des conséquences indésirables qu'il s'agisse de sur-restaurations ou de sous-restaurations. Le premier cas, le plus fréquent, consiste à essayer de remettre l'objet dans son état d'origine. Si ce type de restauration n'est pas véritablement en mesure d'effacer l'action du temps - même si tel est le but recherché -, elle peut par contre effacer les traces matérielles du passage du temps au détriment de la compréhension de l'objet et de son histoire. Le second type d'intervention, beaucoup plus rare, tente non pas de remonter le temps, mais de l'arrêter, interrompant ainsi artificiellement la vie de l'objet.

Sans aucun doute, la décision de faire une coupe de l'objet ou de le faire fonctionner - et par conséquent de provoquer plus d'usure et de dommages -, peut mettre en péril des marques signifiantes que le temps a laissées, aussi dans la majeure partie des cas cela devrait-il être déconseillé. Si cet argument semble concis et logique, il s'appuie cependant sur la certitude que l'objectif premier de la restauration est de conserver une trace historique et que celle-ci est de nature purement matérielle. Si, comme l'avance Mann', ces hypothèses sont invalidées, c'est l'éthique de conservation qu'il faudrait alors modifier.

Il serait sans doute plus fructueux d'adopter une position médiane. S'il s'agit d'un objet fabriqué en série - c'est-à-dire s'il en existe encore plus d'un exemplaire -, ou si l'importance de l'objet en soi est minimale, il pourrait être justifié d'entreprendre une restauration en profondeur et d'essayer de faire fonctionner à nouveau l'instrument. Par contre, s'il s'agit d'objets très spécifiques d'importance historique, ou de pièces uniques, il serait préférable de faire de la conservation préventive et d'utiliser des répliques pour permettre au public d'en comprendre l'usage et le fonctionnement.

Toute procédure, tous travaux ou toute action susceptibles d'altérer le plan, la forme, l'apparence, le style d'origine, ainsi que les principes de base ou des détails de l'objet devraient être évités. En particulier, tout ajout devrait être scrupuleusement proscrit pour des raisons d'authenticité. Les seules parties qui puissent être remplacées par des éléments neufs sont soit celles dont on est sûr de connaître la forme, la dimension, la position, les mouvements, l'apparence, etc. de sorte que les parties nouvelles correspondent exactement à l'original, soit celles qu'il faut changer pour des questions évidentes de sécurité du public.

Plus récemment, Rolland-Villemot ${ }^{10}$ présenta les conclusions d'un groupe de travail mis en place en 1996 par la direction des Musées de France pour discuter et réfléchir à la 
définition de méthodologies de conservation et de restauration des objets techniques et industriels. Le groupe de travail identifia huit points à considérer préalablement à toute action de conservation ou de restauration de ce type d'objets :

- Le statut de l'objet. Est-ce un objet unique conservé en un seul exemplaire? un prototype ? une maquette? un objet de série? un objet pédagogique? Le statut de l'objet est déterminant dans le choix du parti pris de restauration.

- Le diagnostic. Il est nécessaire d'établir un constat précis de l'état de conservation et de l'intégrité de l'objet.

- Un projet scientifique et culturel autour de l'objet. Il est notamment essentiel que l'objet à restaurer soit préalablement étudié et compris sous tous ces aspects, quitte ensuite à privilégier pour des raisons muséographiques ou techniques un aspect spécifique.

- La constitution d'une équipe pluridisciplinaire qui regroupe toutes les compétences nécessaires à un projet autour d'un objet industriel.

- La rédaction d'une étude préalable. Il faut définir avant toute opération de restauration les objectifs de celle-ci. Cette étude doit notamment comprendre une approche historique et technique, un constat d'état, des propositions de restauration.

- La rédaction d'un cahier des charges qui doit établir toutes les interventions sur l'objet et qui doit également prévoir les mesures d'entretien et de maintenance de l'objet.

- Le suivi et l'encadrement des opérations avec une définition précise des rôles de chacun (maîtrise d'ouvrage et maîtrise d'œuvre).

- La documentation à réunir en vue d'une connaissance exacte de l'objet et pour un suivi des opérations de restauration.

Pour Bonsanti ${ }^{11}$, par comparaison avec d'autres types de restauration, celle des instruments scientifiques nécessite :

- un haut niveau de formation dans le domaine des matériaux - bois, grande variété de métaux et d'alliages, verre, etc. ;

- des connaissances en histoire et une sensibilité particulière à l'approche historique pour des interactions fructueuses avec les historiens des sciences et pour l'acquisition d'une connaissance approfondie des spécificités de l'objet ;

- une familiarité avec les techniques de construction dans le domaine de la mécanique de précision, s'appuyant sur l'examen de différents exemples du même type et sur des contacts avec des scientifiques ;

- une grande dextérité manuelle de sorte que, quelles que soient les pièces manquantes, celles-ci puissent si nécessaire être reconstruites de façon historiquement appropriée et techniquement réussie.

20 L'ensemble de ces qualifications implique nécessairement la constitution d'une équipe multidisciplinaire, étant donné qu'a priori une seule personne ne peut toutes les posséder.

21 Enfin, tous les travaux de restauration doivent pouvoir être détectés, pas nécessairement à première vue ou à l'œil nu, mais par un observateur non expert laissé cinq minutes seul avec l'objet et équipé d'une loupe ${ }^{12}$. 


\section{Le MAST et sa politique de restauration} de la Technologie, le Museu de Astronomia e Ciências Afins (musée d'Astronomie et des sciences connexes, MAST) a pour mission principale la conservation et la sauvegarde des collections d'instruments scientifiques du Brésil, et notamment de celle de l'Observatoire national de Rio de Janeiro. Physiquement hébergé sur le site de l'observatoire, le MAST y occupe un certain nombre d'édifices du début du xxe siècle et ce sont précisément les instruments qui ont été collectés sur ce site qui lui confèrent son statut de musée des sciences et des techniques. L'ensemble des bâtiments historiques ainsi que les instruments qui s'y trouvaient ont été protégés au titre de l'IPHAN ${ }^{13}$ par une loi fédérale adoptée en 1986. Récemment restauré, le bâtiment principal du musée abrite les espaces techniques. C'est dans ce bâtiment qu'est conservée la majeure partie de la collection des instruments scientifiques historiques.

Avec ses 2000 objets, dont environ 1600 proviennent de l'ancien Observatoire national, le MAST possède une collection extrêmement diversifiée qui a sa place parmi les grandes collections de ce genre ${ }^{14}$. Les instruments provenant de l'observatoire furent utilisés pour des tâches de service et des recherches de grande importance pour le pays : détermination et distribution de l'heure officielle au Brésil, prévision du temps, observation de phénomènes astronomiques, délimitation des frontières, établissement de la carte magnétique du territoire brésilien, etc. Pour la plupart, ces instruments remontent au xixe et au début du xxe siècle, tandis que les plus intéressants sur le plan esthétique - comme le quadrant de Sisson et le théodolite de G. Adams - datent du xviiie siècle. Typiques des institutions auxquelles ils appartenaient, de nombreux objets relèvent de l'astronomie, de la topographie, de la géodésie, de la géophysique, de la météorologie, du temps et des mesures optiques. Certains éléments de la collection relèvent aussi d'autres domaines dont l'électricité, le magnétisme et la chimie. d'explication. Le MAST conserve non seulement des instruments présents dans des institutions similaires ou des musées (télescopes, théodolites, cercles méridiens, horloges de précision, magnétomètres, instruments de météorologie, etc.), mais également des pièces très particulières et très rares. Parmi ces dernières, une machine à prédire les marées de Kelvin, un analyseur de Henrici, un instrument de Salmoiraghi pour la détermination de l'équation personnelle, des instruments pour placer les fils des réticules ainsi que d'autres instruments spécialisés. Enfin, l'un au moins des instruments de la collection du MAST est unique : un altazimut de la fin du xixe siècle inventé par Emmanuel Liais ${ }^{15}$ et fabriqué dans les ateliers d'Hermida Passos à Rio de Janeiro. Cet altazimut fut d'ailleurs primé lors de diverses expositions organisées au Brésil ou en Europe.

Il est intéressant de souligner que l'étude et l'analyse de ces instruments permettent à la fois d'évaluer la potentialité scientifique de chacun d'eux et de déduire l'évolution au cours du temps - ou, dans certains cas, la stagnation - des méthodes de travail des astronomes. La présence de ces instruments témoigne aussi de ce que fut l'institution " observatoire de Rio de Janeiro ", et notamment de quels objectifs elle fut porteuse et des activités scientifiques qu'elle entreprit.

Environ $98 \%$ des objets appartenant à la collection du MAST sont dans un état de conservation bon ou satisfaisant. Les $2 \%$ restants correspondent à 40 objets dont l'état 
a été évalué en termes de restauration. Pour l'essentiel, les instruments de la collection ne nécessitent qu'un nettoyage périodique auquel il est procédé environ une fois tous les deux ans.

Figure 1

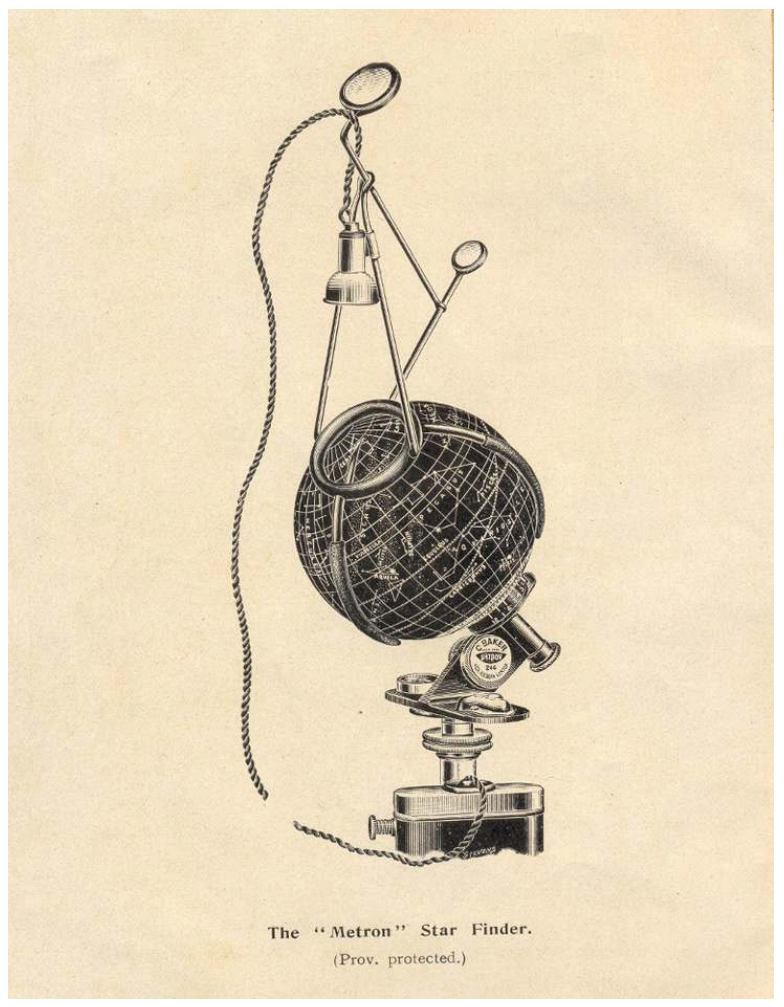

Globe pour identifier les étoiles (star finder). Planche extraite de la notice technique THE METRON STAR FINDER, C. BAKER, 244, High Holborn, London, Scientific Instruments Manufacturer. Londres, vers 1908, 17 pages, 1 illustration.

Archives du Museu de Astronomia e Ciências Afins, Rio de Janeiro, Brésil @ MAST.

En ce qui concerne la restauration, quatre instruments ont déjà fait l'objet d'interventions, deux provenant de constructeurs français - un théodolite des frères Brunner et le grand cercle méridien de Gautier -, et deux de constructeurs anglais - une lunette équatoriale de $32 \mathrm{~cm}$ de diamètre de Thomas Cooke \& Sons et un globe pour identifier les étoiles (star finder) de Metron du fabricant londonien C. Baker ${ }^{16}$ (fig. $\left.\mathbf{n}^{\circ} \mathbf{1}\right)$. Les trois premiers remontent au xixe siècle, tandis que le quatrième date du début xxe siècle. La sélection de ces quatre instruments a été effectuée selon les critères suivants :

- potentiel historique des pièces, utilisées à l'observatoire à l'occasion de travaux de recherche importants;

- notoriété des fabricants, connus pour avoir produit des instruments scientifiques de qualité nécessitant de grandes qualifications techniques ;

- détérioration des surfaces métalliques, très oxydées et dont le vernis laqué original était en partie manquant ;

- pour le théodolite et le cercle méridien, absence de certains éléments de l'instrument, ce qui allait permettre de procéder à l'une des opérations les plus critiques de la restauration, à savoir le remplacement de parties manquantes. 
Pour chacune de ces opérations, la procédure suivie fut identique. Tout d'abord, des recherches historiques furent entreprises afin de collecter un maximum d'informations sur les pièces à restaurer, y compris sur leur fonctionnement. Au cours de l'étape suivante, les instruments furent complètement démontés et leurs éléments nettoyés mécaniquement. Les parties à restaurer furent alors séparées des autres. Les produits de la corrosion furent éliminés de façon uniquement mécanique, puis les surfaces nettoyées à l'alcool éthylique et au trichloréthylène. Enfin, celles-ci furent pour la plupart recouvertes soit de laque soit de peinture, suivant leur revêtement original.

29 Avant d'exposer de façon plus approfondie l'opération de restauration du plus emblématique de ces quatre instruments - le cercle méridien Gautier - nous retraçons brièvement l'histoire institutionnelle de l'observatoire de Rio de Janeiro, puis nous évoquons le contexte de la commande de cet instrument prestigieux ainsi que les différentes étapes de son histoire en tant qu'objet, histoire par ailleurs commune à nombre d'instruments scientifiques de par le monde : en caisse, en fonction, obsolète, démonté, oublié...

\section{Brève histoire de l'observatoire de Rio de Janeiro et de son cercle méridien Gautier}

\section{Les premières décennies : un établissement à l'usage des militaires}

Alors que sous l'impulsion du marquis de Pombal (1699-1782) de nombreuses activités scientifiques s'étaient développées au Portugal durant la seconde moitié du xviiie siècle, au Brésil la situation était bien différente. Les quelques tentatives d'enseignement entreprises par des jésuites tout comme les académies créées en diverses villes à partir du xviie siècle étaient en effet restées sans suite ${ }^{17}$. La situation ne commença à changer qu'à partir de 1808 lorsque, fuyant les armées napoléoniennes, la famille royale abandonna Lisbonne pour Rio de Janeiro ${ }^{18}$. Signe de son intérêt pour la science, João VI (1767-1826), régent en 1799 puis roi à la mort de sa mère en 1816, avait d'ailleurs pris soin d'apporter $d u$ Portugal nombre d'instruments scientifiques prestigieux ${ }^{19}$.

31 Soucieux de développer l'enseignement, notamment pour former les cadres nécessaires à l'administration du vaste empire portugais, le futur roi créa rapidement à Rio de Janeiro plusieurs écoles supérieures - dont en 1808 l'Académie de marine et en 1810 l'Académie royale militaire. Dès 1811 des observations astronomiques à finalité utilitaire - essentiellement pour l'établissement de cartes - furent effectuées dans cette dernière. En 1822, deux ans après qu'une révolution pacifique eut ramené João VI au Portugal, Pedro (1798-1834), fils aîné de ce dernier, proclama l'indépendance du Brésil et s'en déclara empereur. Le 15 octobre 1827 dom Pedro Ier décida la création à Rio de Janeiro d'un observatoire astronomique ayant pour mission à la fois d'effectuer des observations astronomiques et météorologiques et d'enseigner l'astronomie aux étudiants des académies militaire et navale ${ }^{20}$. 


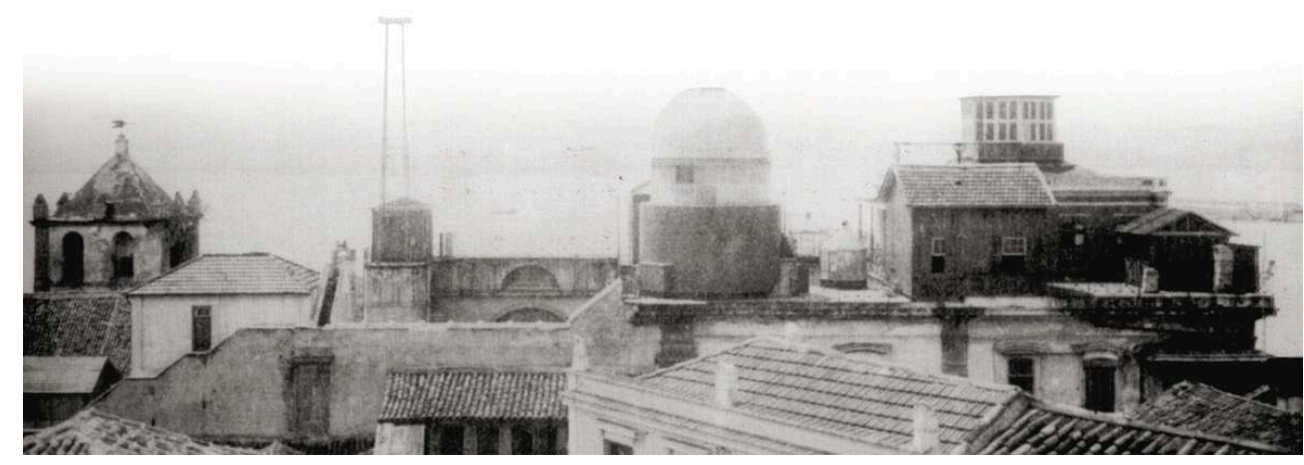

L'observatoire impérial de Rio de Janeiro sur le Morro do Castelo, vue générale depuis l'ouest. Photographie de la seconde moitié du XIXe siècle.

Archives du Museu de Astronomia e Ciências Afins, Rio de Janeiro, Brésil @ MAST.

Tout d'abord établi à l'Académie militaire - renommée École militaire en 1840 au tout début du règne de dom Pedro II (1825-1891) -, l'observatoire devint par décret en 1846 Observatoire impérial de Rio de Janeiro. Il quitta alors l'espace inadapté et exigu dans lequel il était installé et fut transféré sur une élévation naturelle dominant le port de Rio, le Morro do Castelo (fig. $\mathbf{n}^{\circ}$ 2). Site stratégique occupé depuis 1567 par une citadelle militaire, cette colline avait aussi été le siège d'un collège de jésuites. Après l'expulsion de ces derniers de l'empire du Portugal en 1758, les installations du collège avaient abrité l'hôpital militaire de la cour, et l'église que les jésuites avaient entrepris d'y élever avait été abandonnée. De 1846 à 1850, le directeur de l'observatoire, Soulier de Sauve ${ }^{21}$, professeur à l'École militaire sans réelle expérience de l'astronomie, transféra l'observatoire dans cette église inachevée appartenant au ministère de la Guerre $^{22}$. D'après le décret de 1846 l'établissement avait alors pour mission d'effectuer des observations astronomiques et météorologiques, de former les étudiants de l'École militaire et de l'Académie de la marine, et également de publier des éphémérides et de fournir l'heure aux navires du port de Rio de Janeiro ${ }^{23}$. 


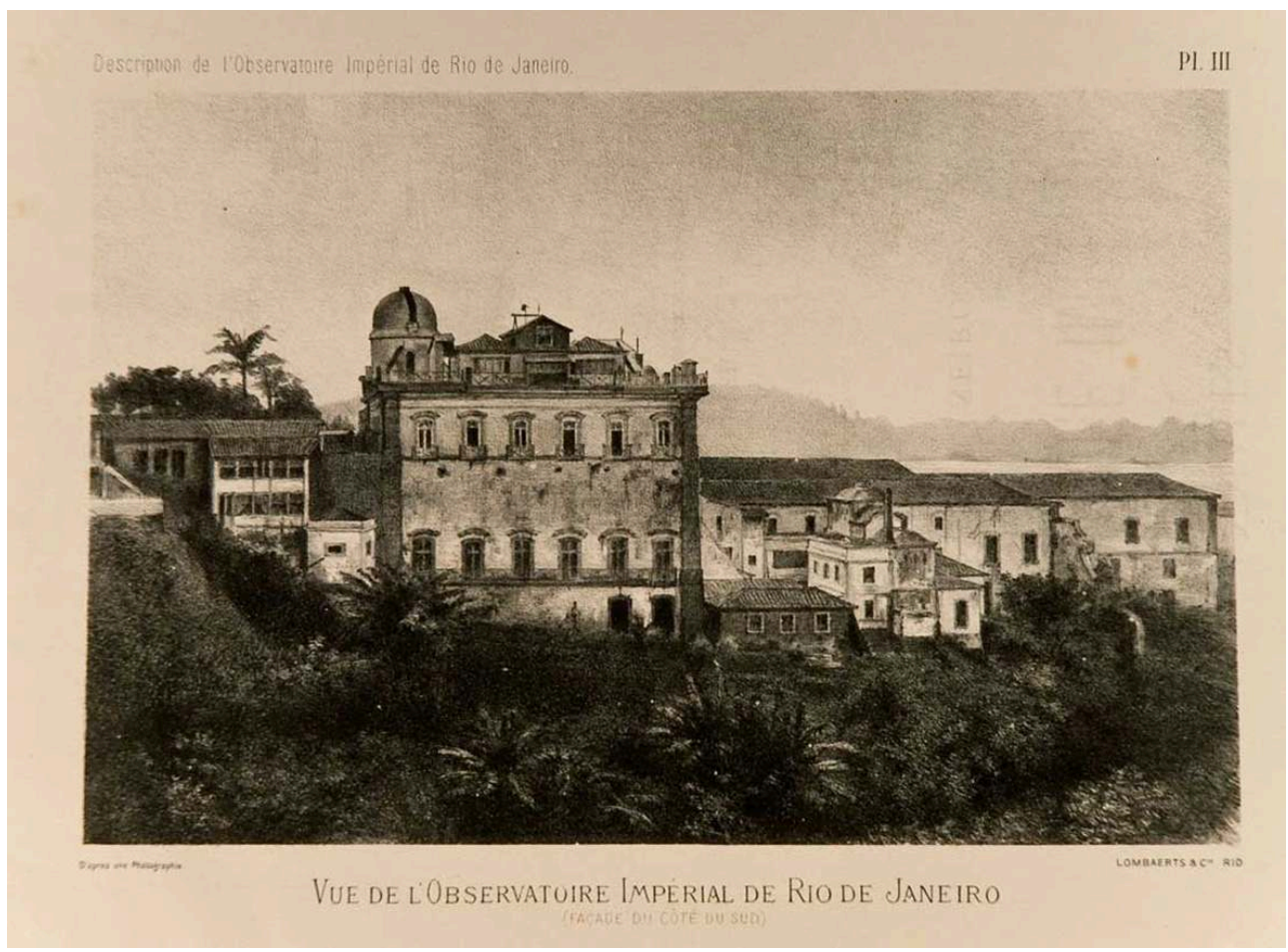

L'observatoire impérial de Rio de Janeiro sur le Morro do Castelo, vue de l'élévation sud. LIAIS, Emmanuel. ANNALES de L'observatoire IMPÉRIAL de RIO de JANEIRo, Tome premier, Rio de Janeiro : Lombaerts \& Cie, 1882, planche III. Repro. Marc Heller, 2008.

Successeur de Soulier de Sauve en 1850, le lieutenant-colonel Antônio Manuel de Mello ( 1795- 1865) ne fut pas beaucoup plus actif dans le domaine de l'astronomie. Il organisa cependant des expéditions au Brésil à l'occasion des éclipses totales de Soleil de 1858 et de 1865. Le plus grand instrument de l'observatoire installé à son époque est une lunette de $10 \mathrm{~cm}$ d'ouverture due au constructeur anglais Dollond ${ }^{24}$. Très régulièrement, de Mello publia également des éphémérides astronomiques destinées à la marine brésilienne et à partir de 1851 des observations météorologiques ${ }^{25}$. Nommé commandant en chef de la cavalerie brésilienne en 1864 au début de la guerre dite du Paraguay (1864-1870), le général de Mello "succomba bientôt aux fatigues de la guerre ${ }^{26}$ à l'âge de 70 ans. Son ancien aide de camp, le capitaine-lieutenant Antônio Joaquim Cruvelo d'Avila lui succéda en 1865, l'année où l'observatoire passa sous la tutelle de l'École centrale qui venait d'être détachée de l'École militaire. Mais jusqu'à la fin de son mandat en 1871, « on n'observa pas plus qu'auparavant $»^{27}$ (fig. $\mathbf{n}^{\circ} \mathbf{3}$ ).

\section{D'un observatoire militaire à un observatoire astronomique}

Une fois la paix revenue, l'empereur dom Pedro II, lui-même passionné d'histoire naturelle et féru d'astronomie, entreprit de réorganiser l'observatoire. En janvier 1871 celui-ci fut détaché par décret de sa tutelle militaire, puis pour la première fois depuis sa création il fut doté d'un directeur astronome. D'origine française, Emmanuel Liais $(1826-1900)^{28}$ avait rencontré l'empereur pour la première fois en 1859 à Olinda, près de Recife $^{29}$. 
Figure 4

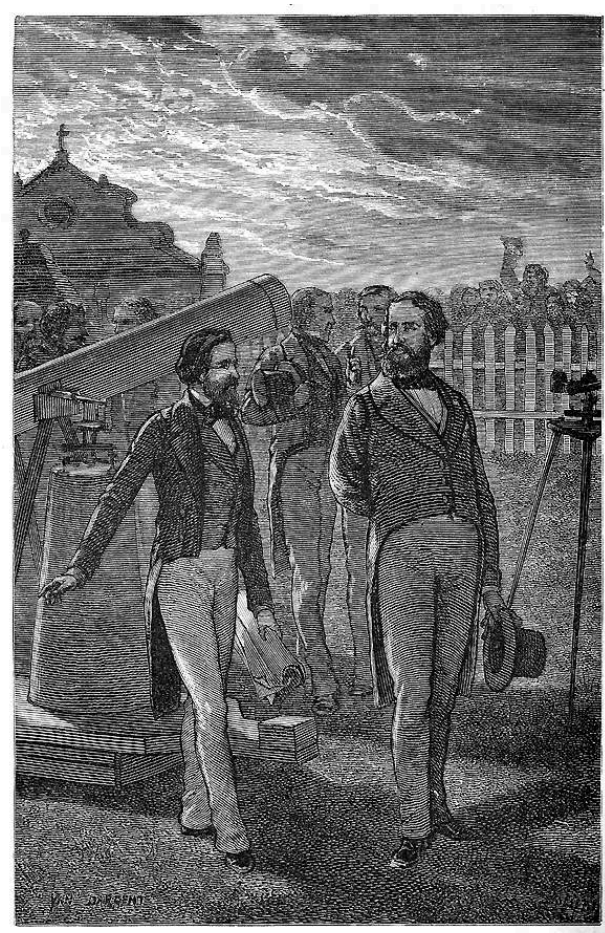

Visite de S. M. l'Empereur du Brésil à l'observatoire d'olinda (p. 2ss)

L'empereur du Brésil et Emmanuel Liais à Olinda, 1859. LIAIS, Emmanuel. L'espace céleste et la nature tropicale : Description physique de l'univers d'après des observations personnelles faites dans les deux hémisphères. Paris : Garnier frères, 1865, page 289. Repro. Marc Heller, 2008. 
Figure 5

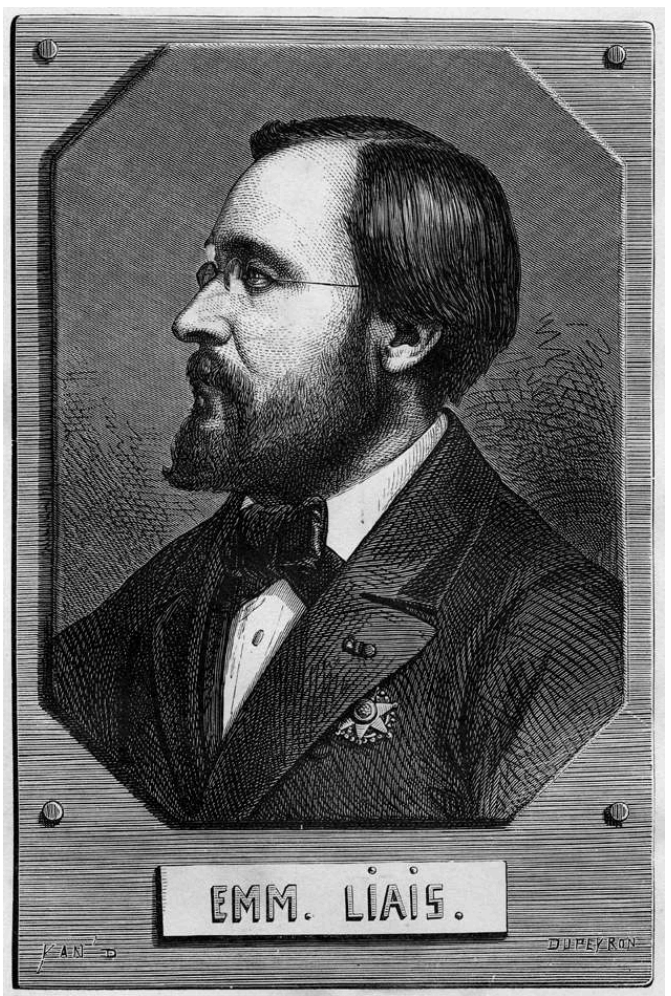

Portrait d'Emmanuel Liais. LIAIS, Emmanuel. L'espace céleste et la nature tropicale : Description physique de l'univers d'après des observations personnelles faites dans les deux hémisphères. Paris: Garnier frères, 1865, page de garde. Repro. Marc Heller, 2008.

Sa femme Margaritha Trovwen (1833-1874) ${ }^{30}$ et lui avaient séjourné au Brésil en tant qu'explorateurs de 1858 à 1864, puis y étaient revenus en 1867. Nommé en 1871, Liais ne prit cependant son poste qu'en 1874, après un séjour en Europe durant lequel il fit construire certains des instruments nécessaires à un observatoire national de premier rang $^{31}$. Preuve du renouveau scientifique de l'astronomie brésilienne, à la demande de l'empereur, un professeur intérimaire de l'École polytechnique de Rio, Francisco Antonio de Almeida, fut envoyé au Japon auprès de l'astronome français Jules Janssen (1824-1907) parti observer le passage de Vénus du 9 décembre $1874^{32}$. L'arrivée de Liais marqua le début d'une période productive au cours de laquelle l'observatoire de Rio présenta des travaux scientifiques à diverses académies européennes. Pendant cette période faste, Liais prépara un descriptif détaillé et illustré de l'observatoire et de ses installations destiné à inaugurer la série des Annales de l'observatoire de Rio de Janeiro - à l'instar de ce qui se faisait dans de nombreux observatoires européens. (fig. $\mathbf{n}^{\circ} \mathbf{6}$, $\left.n^{\circ} 7, n^{\circ} 8, n^{\circ} 9\right)$ 
Figure 6

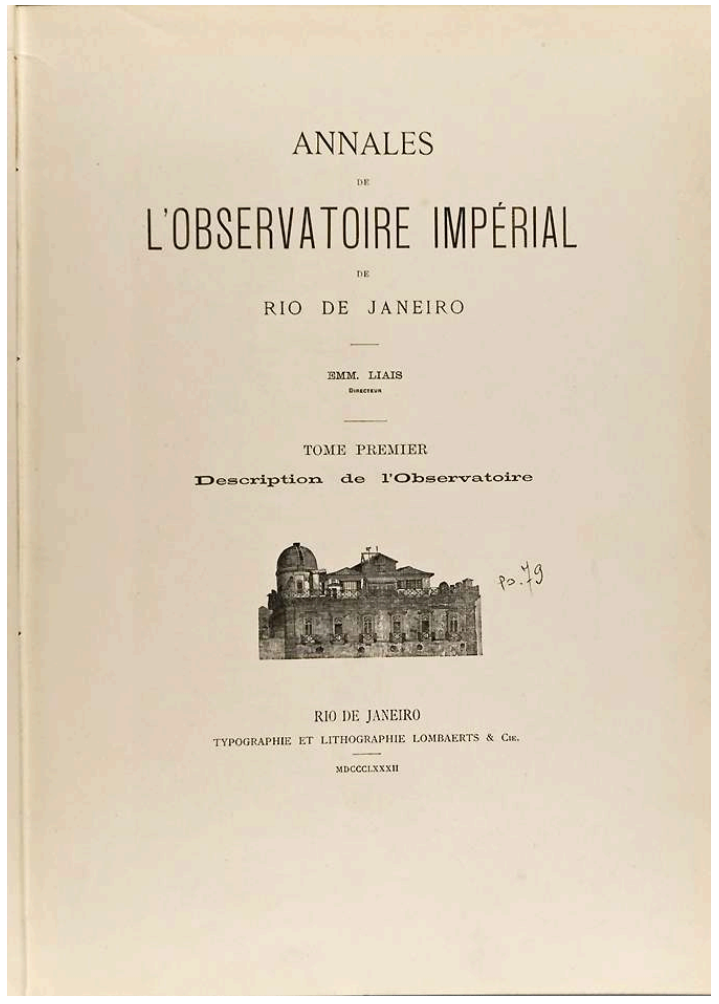

Page de garde du Tome I des Annales de l'observatoire de Rio de Janeiro rédigé par Liais et publié par Cruls après que Liais a quitté le Brésil ; on distingue dans un médaillon une gravure de l'observatoire du Morro do Castelo. LIAIS, Emmanuel. ANNALES de L'OBSERVATOIRE IMPÉRIAL DE RIO DE JANEIRO, Tome premier, Rio de Janeiro : Lombaerts \& Cie, 1882. Repro. Marc Heller, 2008. 
Figure 7

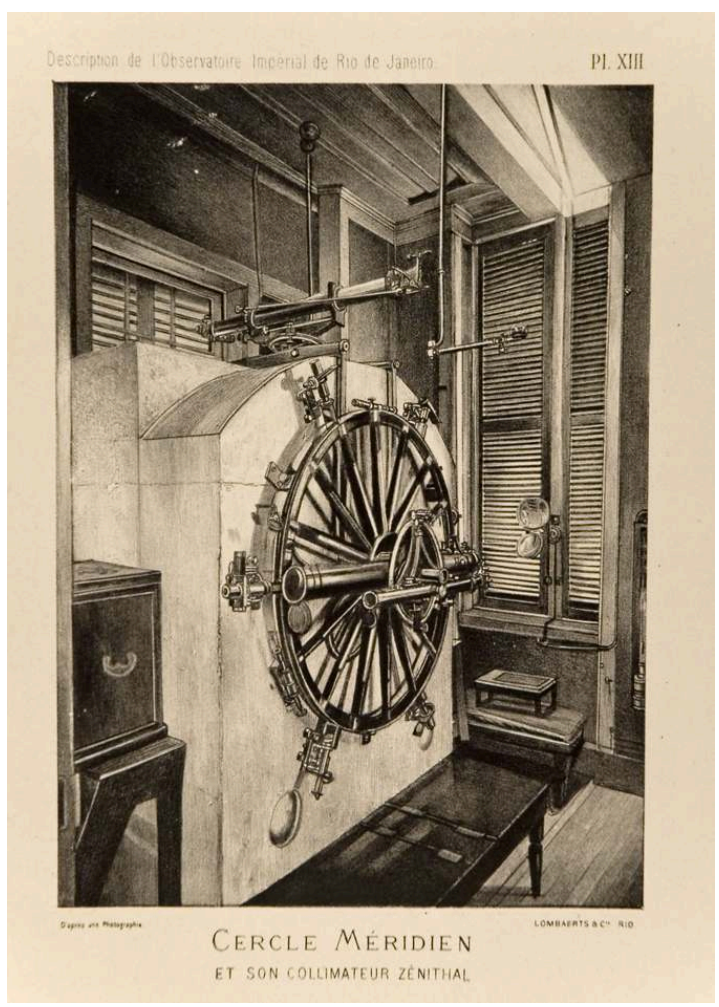

Cercle mural Dollond et son collimateur zénithal sur leur pilier en pierre (vue de face), observatoire impérial de Rio de Janeiro, site de Morro do Castelo. LIAIS, Emmanuel. ANNALES DE L'oBservatoire IMPÉriAL DE RIO De JANEIRo, Tome premier, Rio de Janeiro : Lombaerts \& Cie, 1882, planche XIII. Repro. Marc Heller, 2008. 
Figure 8

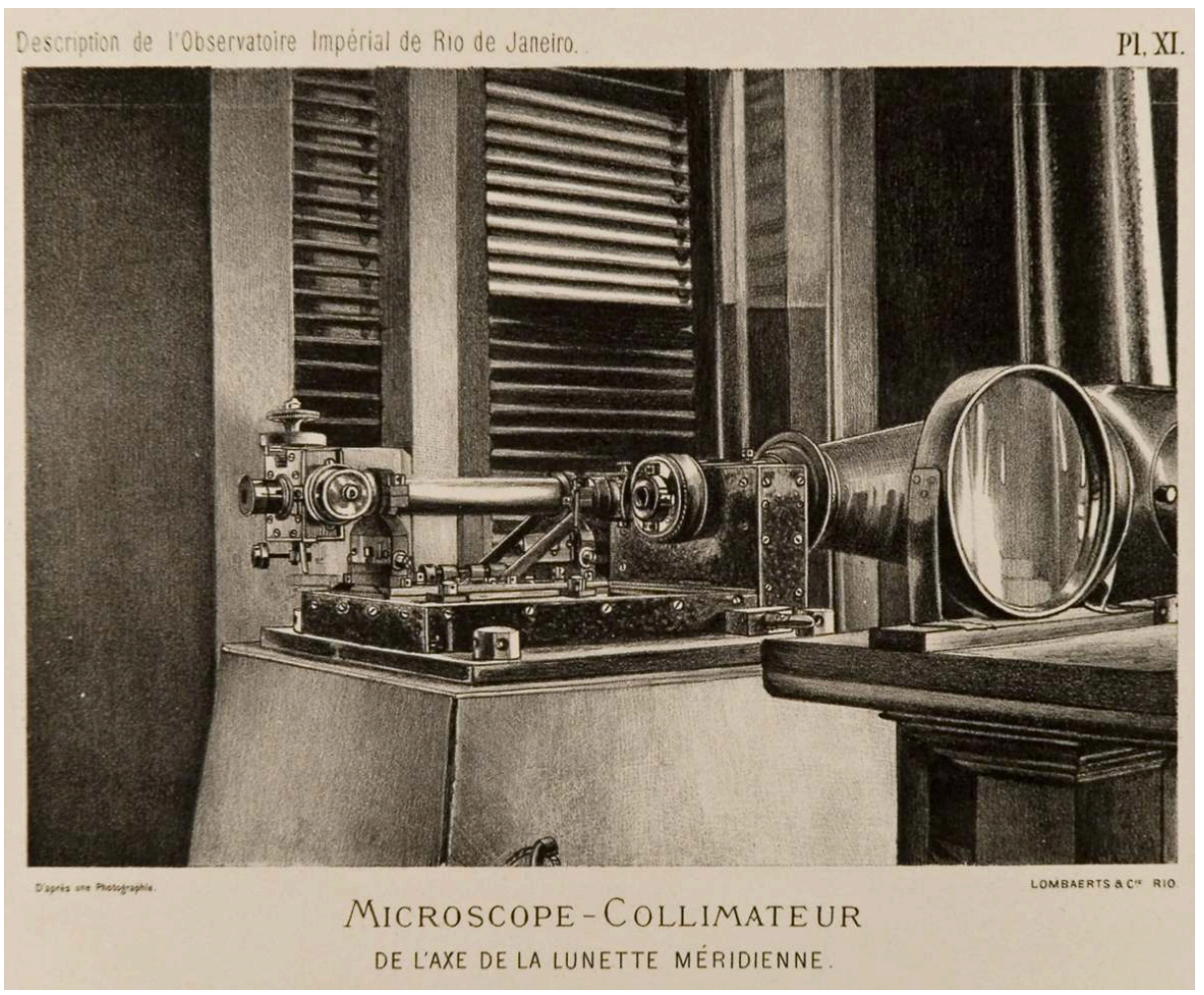

L'un des piliers de la lunette méridienne Dollond montrant le microscope-collimateur placé sur l'axe de la lunette, observatoire de Rio de Janeiro, site de Morro do Castelo. LIAIS, Emmanuel. ANNALES DE L'observatoire IMPÉRIAL de RIO de JANEIRo, Tome premier, Rio de Janeiro : Lombaerts \& Cie, 1882, planche XI. Repro. Marc Heller, 2008. 


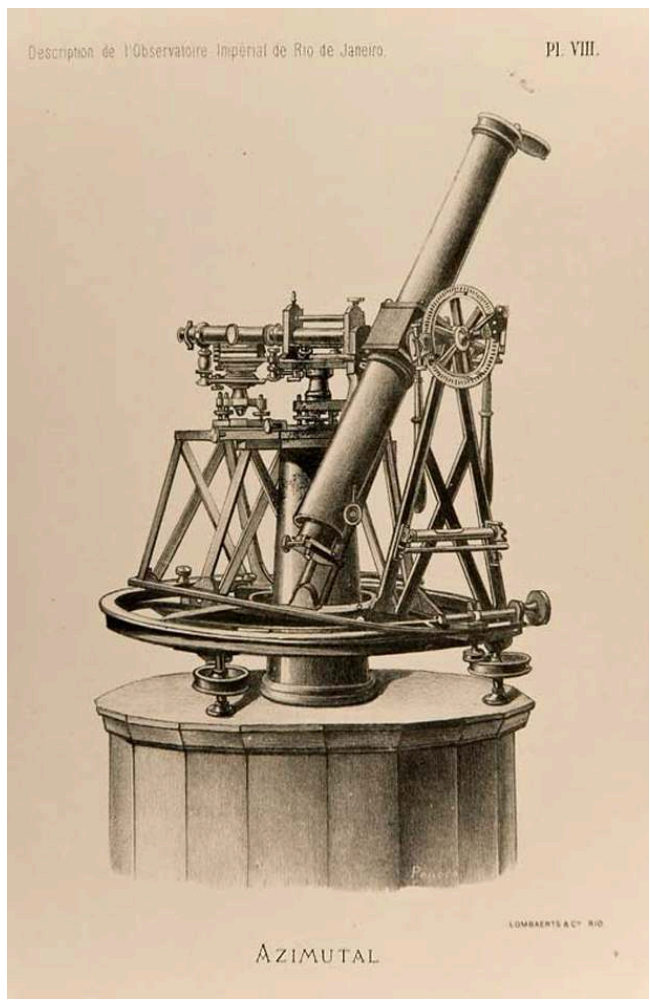

Instrument azimutal construit dans les ateliers de José Maria dos Reis sur les plans de Liais ; cet instrument qui était en service en 1875 a notamment permis d'établir la latitude absolue de l'observatoire de Morro do Castelo. ANDRÉ, Charles, ANGOT, André. L'ASTRONOMIE PRATIQUE : Les OBSERVATOIRES EN EUROPE ET EN AMÉRIQUE, QUATRIÈME PARTIE, OBSERVATOIRES DE L'AMÉRIQUE dU SUD. Paris : Gauthier-Villars, 1881, Figure 6, p. 35.

L'Observatoire impérial fut d'ailleurs considéré à cette époque comme l'une des meilleures institutions scientifiques du Brésil ${ }^{33}$. Cependant, devenu un haut lieu d'activités scientifiques contribuant à l'institutionnalisation de la science au Brésil, l'établissement délaissait les activités destinées à l'Armée et à la Marine, à savoir l'enseignement de l'astronomie et «l'astronomie pratique ». Peu à peu la direction de Liais suscita de graves controverses personnelles et scientifiques, ce qui aboutit à son départ en $1881^{34}$.

Louis Cruls (1848-1908), ingénieur et astronome d'origine belge et principal collaborateur de Liais, se vit alors confier la direction de l'observatoire national ${ }^{35}$. Durant sa direction, l'observatoire fut invité à participer à une opération internationale d'envergure: l'entreprise de la Carte photographique du ciel, lancée en 1887 par le contre-amiral Mouchez (1821-1892), alors directeur de l'observatoire de Paris. La lunette photographique mise au point à Paris pour l'opération fut commandée par l'empereur, mais divers obstacles, dont les troubles politiques qui en 1889 accompagnèrent la proclamation de la République, empêchèrent l'observatoire de contribuer aux travaux de la Carte du ciel au côté des dix-sept autres établissements ayant décidé d'y participer ${ }^{36}$. L'instrument acquis ne fut donc jamais installé sur le site qu'avait proposé dom Pedro II en vue du transfert de l'observatoire, un domaine de 40 hectares situé dans la Fazenda impériale de Santa $\mathrm{Cruz}^{37}$.

Peu après l'avènement du régime républicain en 1889, l'observatoire impérial passa sous la tutelle du ministère de la Guerre et changea de nom pour devenir simplement 
observatoire de Rio de Janeiro. Dans le rapport annuel de 1891 de la nouvelle tutelle de l'observatoire, il est fait mention à la fois de l'abandon d'un projet de déménagement de l'établissement au Morro de Santa Thereza (Nova Cintra), des études en cours pour trouver un site du côté de Petrópolis et des instruments astronomiques commandés en Europe : une lunette équatoriale photographique (la lunette de la Carte du ciel) et un cercle méridien.

\section{Un cercle méridien Gautier à l'observatoire de Rio de Janeiro}

Né à la fin du xviie siècle de l'association de deux instruments - lunette des passages et cercle mural - en un seul, le cercle méridien possède un axe horizontal orienté estouest dont les extrémités reposent sur deux piliers très stables. La lunette ainsi que le(s) cercle(s) ${ }^{38}$ de lecture qui en est (sont) solidaire(s) sont orientés précisément dans le plan nord-sud. Associé à un régulateur astronomique, cet instrument permet la mesure simultanée de l'instant de passage d'un astre dans le plan méridien et de sa hauteur audessus de l'horizon. Ces deux données permettent ensuite de déterminer les coordonnées célestes des astres (ascension droite et déclinaison) avec une grande précision et de produire des catalogues.

L'instrument méridien à cercle(s) n'équipera systématiquement la plupart des observatoires d'État qu'à partir du xixe siècle ${ }^{39}$. Après l'observatoire de Pulkovo équipé dès sa fondation en 1835 par Wilhelm Struve (1793-1864) d'un grand cercle méridien Repsold dont la lunette a un objectif de $15 \mathrm{~cm}$ de diamètre, c'est celui de Greenwich qui en 1850, sous l'impulsion de l'astronome royal George Airy (1801-1892), installa un imposant cercle méridien de $20,5 \mathrm{~cm}$ de diamètre dû à Troughton et Simms. En 1862, James Gilliss (1811-1865), directeur de l'observatoire naval de Washington, commanda au constructeur allemand Pistor et Martins un cercle méridien de $23 \mathrm{~cm}$ de diamètre qui sera installé en 1866. Un instrument aussi puissant que celui de Washington, commandé à Secrétan ${ }^{40}$, fut installé par Leverrier dans la salle des méridiens du bâtiment Perrault de l'observatoire de Paris en 1864. Mais cet instrument ne donnant pas entièrement satisfaction, dès 1873 Leverrier passa commande au constructeur parisien Wilhelm Eichens (1818-1894) d'un nouveau cercle méridien qui, mis en service en 1877, s'avéra excellent. Peu après, l'État français passa commande à Eichens, puis à son successeur Paul Gautier (1842-1909) ${ }^{41}$, d'une série de cercles méridiens destinés à équiper la totalité des observatoires de province.

\section{Tableau comparatif des cercles méridiens français installés dans le monde de 1875 à 1900}

\begin{tabular}{|l|l|l|l|l|l|}
\hline Observatoire & Date & Constructeur & Diamètre des cercles & Ouverture & Distance focale \\
\hline Lima (Pérou) & $1875(?)$ & Eichens & $1 \mathrm{~m}$ & $19 \mathrm{~cm}$ & $2,35 \mathrm{~m}$ \\
\hline Paris & 1877 & Eichens & $1 \mathrm{~m}$ & $19 \mathrm{~cm}$ & $2,32 \mathrm{~m}$ \\
\hline Marseille & 1878 & Eichens & $1 \mathrm{~m}$ & $19 \mathrm{~cm}$ & $2,30 \mathrm{~m}$ \\
\hline Lyon & 1879 & Eichens & $80 \mathrm{~cm}$ & $15 \mathrm{~cm}$ & $2 \mathrm{~m}$ \\
\hline
\end{tabular}




\begin{tabular}{|l|l|l|l|l|l|}
\hline Hendaye & 1880 & Eichens & $70 \mathrm{~cm}$ & $15 \mathrm{~cm}$ & $2 \mathrm{~m}$ \\
\hline Bordeaux & 1881 & Eichens & $1 \mathrm{~m}$ & $19 \mathrm{~cm}$ & $2,32 \mathrm{~m}$ \\
\hline Besançon & 1885 & Gautier & $1 \mathrm{~m}$ & $19 \mathrm{~cm}$ & $2,37 \mathrm{~m}$ \\
\hline Nice & 1887 & Brunner & $80 \mathrm{~cm}$ & $20 \mathrm{~cm}$ & $3,20 \mathrm{~m}$ \\
\hline Alger & 1888 & Gautier & $1 \mathrm{~m}$ & $19 \mathrm{~cm}$ & $2,40 \mathrm{~m}$ \\
\hline Toulouse & 1890 & Gautier & $1 \mathrm{~m}$ & $19 \mathrm{~cm}$ & $2,30 \mathrm{~m}$ \\
\hline Rio de Janeiro (Brésil) & 1890 & Gautier & $1 \mathrm{~m}$ & $19 \mathrm{~cm}$ & $2,35 \mathrm{~m}$ \\
\hline La Plata (Argentine) & 1890 & Gautier & $1 \mathrm{~m}$ & $22 \mathrm{~cm}$ & $2,80 \mathrm{~m}$ \\
\hline Athènes (Grèce) & 1899 & Gautier & $1 \mathrm{~m}$ & $16 \mathrm{~cm}$ & $2,30 \mathrm{~m}$ \\
\hline Tokyo (Japon) & Vers 1900 & Gautier & $1 \mathrm{~m}$ & $22 \mathrm{~cm}$ & $3,10 \mathrm{~m}$ \\
\hline
\end{tabular}

41 Lors du congrès de la Carte du ciel en 1887, Cruls ne manqua sans doute pas de visiter le fameux cercle méridien Eichens dans son abri isolé du jardin de l'observatoire de Paris. Et il discuta probablement avec ses collègues à la fois des autres cercles méridiens de Eichens installés à Marseille, Lyon et Hendaye, et de ceux commandés à Gautier pour Alger, Besançon, Bordeaux et Toulouse. Il n'est donc pas étonnant que ce soit à Gautier, et non à son concurrent allemand Repsold, que l'observatoire de Rio de Janeiro ${ }^{42}$ ait passé commande d'un grand instrument astrométrique ${ }^{43}$. Et ce d'autant que l'équatorial photographique destiné à la participation du Brésil à l'opération de la Carte du ciel était également commandé à Gautier. (fig. $\mathbf{n}^{\circ} \mathbf{1 0}, \mathbf{n}^{\circ} \mathbf{1 1}$ ) 
Figure 10

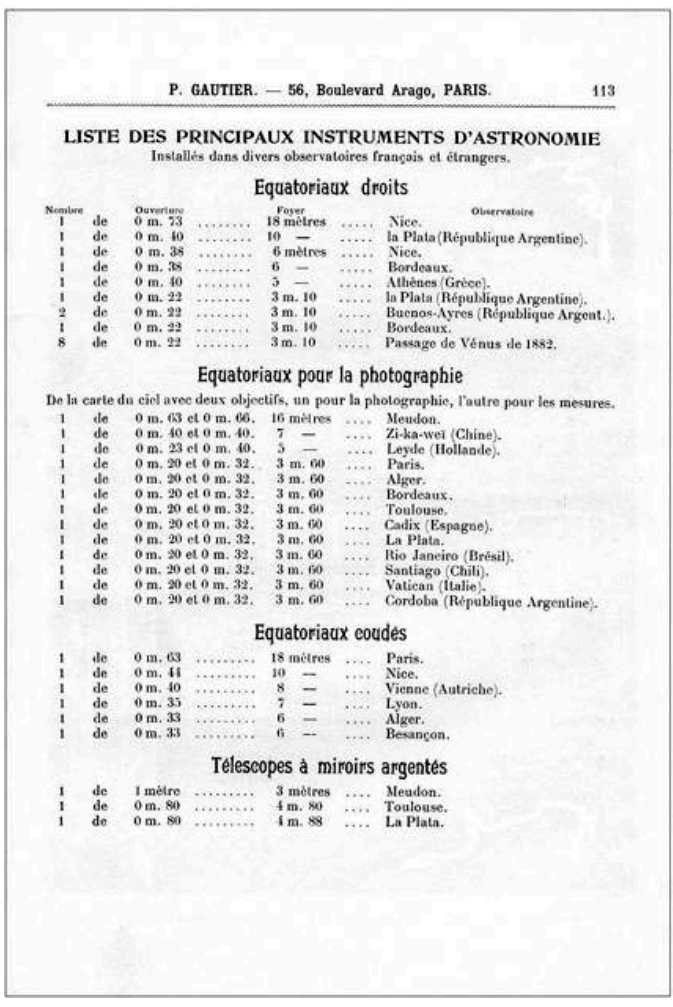

Liste des principaux instruments d'astronomie installés dans des observatoires français et étrangers par Paul Gautier avant 1901. SYNDICAT DES CONSTRUCTEURS EN INSTRUMENTS D'OPTIQUE \& DE PRÉCISION. LIINDUSTRIE FRANÇAISE des INSTRUMENTS DE PRÉCISION, 1901-1902. CATALOgUE. FaCsimilé, Paris : Alain Brieux, 1980, page 113. 
Figure 11

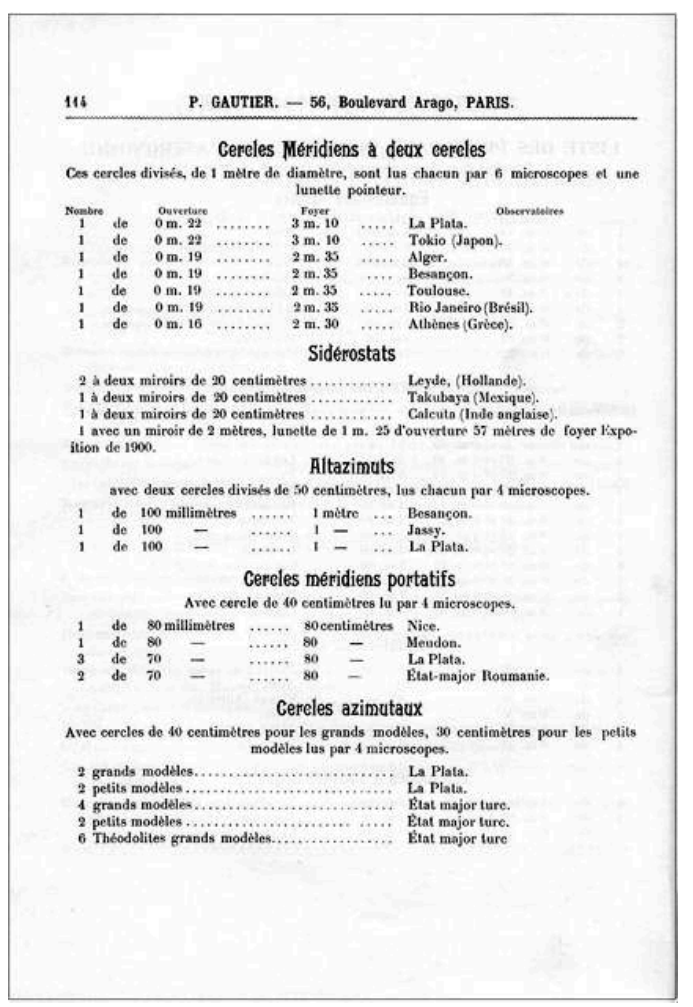

Liste des principaux instruments d'astronomie installés dans des observatoires français et étrangers par Paul Gautier avant 1901. SYNDICAT DES CONSTRUCTEURS EN INSTRUMENTS D'OPTIQUE \& DE PRÉCISION. L'INDUSTRIE FRANÇAISE des INSTRUMENTS dE PRÉcision, 1901-1902. CATALOgUe. Facsimilé, Paris : Alain Brieux, 1980, page 114.

Avec sa lunette de $19 \mathrm{~cm}$ d'ouverture et de 2,40 m de focale et ses deux cercles de $1 \mathrm{~m}$ de diamètre, le cercle méridien de Rio fait partie des grands instruments astrométriques construits en cette fin du xixe siècle. Achevé et livré par Gautier en $1893^{44}$, cinq ans plus tard l'instrument était cependant toujours en caisse ${ }^{45}$.

Pourquoi ? Tout d'abord parce qu'au cours des années 1890 l'observatoire se trouva explicitement sous la tutelle du gouvernement et que Cruls fut mobilisé pour mener à bien diverses expéditions géodésiques ${ }^{46}$. Et puis parce que l'observatoire n'avait toujours pas quitté le Morro do Castelo. Depuis l'implantation de l'établissement sur cette élévation du port de Rio en 1846, le choix du site avait fait l'objet de nombreuses critiques. Des rapports rédigés par les directeurs successifs avaient régulièrement souligné l'instabilité des terrains - formés de gneiss en décomposition - et insisté sur l'impossibilité qui en découlait d'y installer des instruments de grande dimension ${ }^{47}$. Et ils n'avaient pas manqué de dénoncer le préjudice ainsi porté aux activités de l'observatoire. Un demi-siècle plus tard, le site se trouvait de plus au cœur d'une ville en plein essor urbanistique.

En 1900 Cruls décida pourtant de sortir l'instrument de ses caisses et de l'installer au Morro do Castelo dans un abri provisoire en bois ${ }^{48}$. Mais les conditions d'utilisation se révélèrent si défavorables que l'instrument non seulement ne put jamais fonctionner correctement, mais qu'il subit des dommages et qu'il fut nécessaire de procéder à certaines réparations. Aucun résultat ne fut donc obtenu avec ce prestigieux 
instrument. Et il fallut attendre encore quelques années avant que la décision de quitter le Morro do Castelo ne soit effective.

\section{Un nouveau site pour l'observatoire de Rio de Janeiro}

La concomitance au début du xxe siècle de facteurs politiques et de facteurs urbanistiques contribua certainement à dénouer une situation bloquée depuis des décennies. En 1909, Henrique Morize (1860-1930), ingénieur et géographe d'origine française, succéda officiellement à Cruls mort l'année précédente mais malade depuis 1902. Si Morize réussit à convaincre le gouvernement de déménager l'observatoire, il ne fut pas consulté pour le choix du site, sélectionné contre son gré non pour ses qualités astronomiques, mais pour sa proximité avec le port. La décision de transférer l'observatoire sur la colline de São Januário, située dans le quartier alors aristocratique de São Cristovão, fut accompagnée de changements institutionnels : l'établissement devint «Observatoire national », nom toujours en vigueur ${ }^{49}$. Commencés en 1913, les travaux du nouvel ensemble architectural furent achevés en 1920 et l'année suivante l'observatoire quitta enfin le Morro do Castelo pour São Cristovão $0^{50}$. (fig. $\mathbf{n}^{\circ}$ 12)

Figure 12

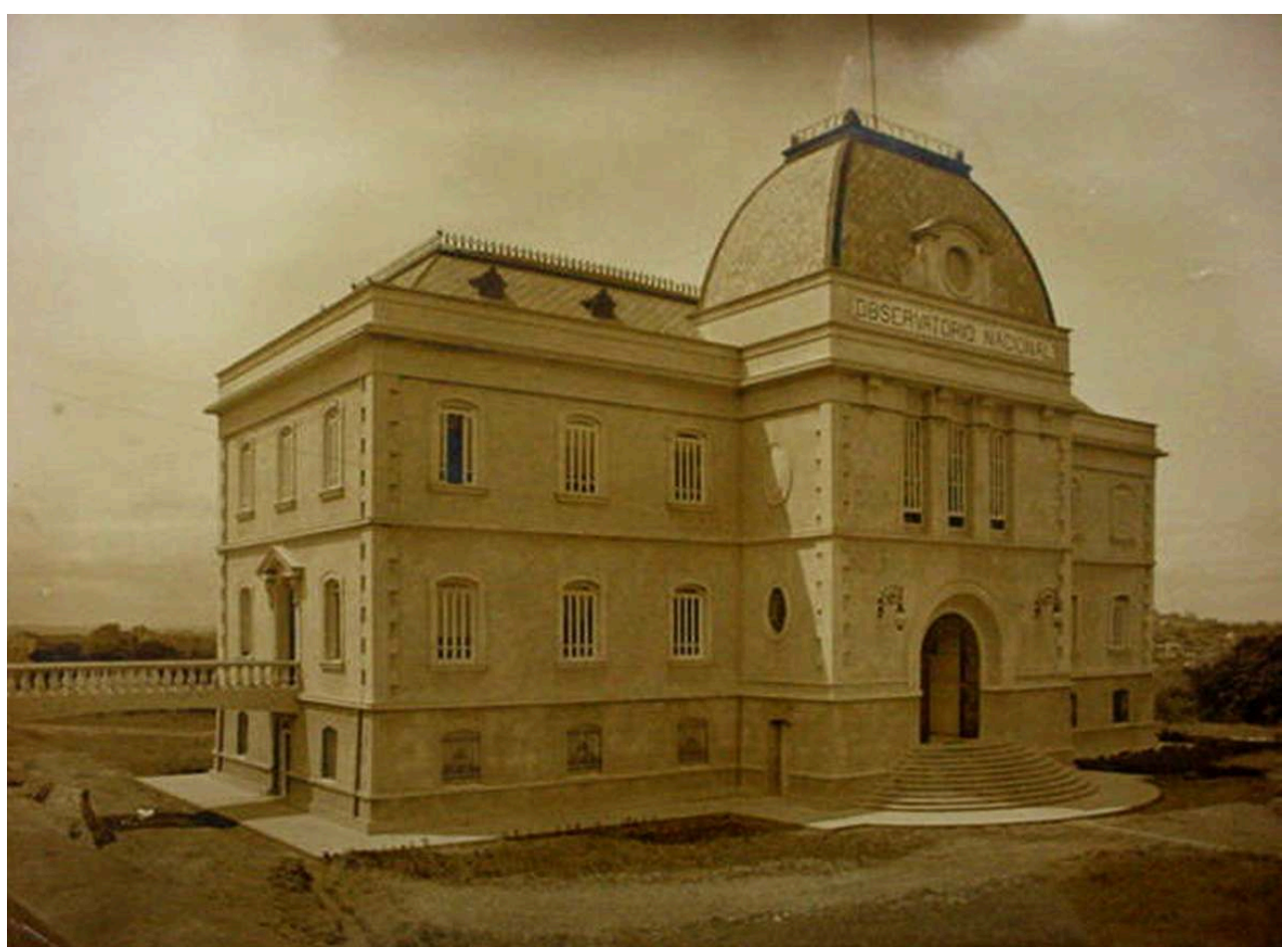

Le bâtiment principal de l'Observatoire national nouvellement implanté sur la colline de São Januário, vue générale en 1922

Archives du Museu de Astronomia e Ciências Afins, Rio de Janeiro, Brésil @ @ MAST.

Les missions incombant à l'établissement comprenaient diverses activités de service et de recherche, parmi lesquelles la détermination officielle du temps pour l'ensemble du Brésil, les prévisions météorologiques, les éphémérides astronomiques, la démarcation des frontières du pays, l'observation systématique des éclipses depuis le territoire national, la cartographie magnétique du sol brésilien, etc. ${ }^{51}$. À cette époque, à nouveau 
contre le gré de Morize, l'établissement fut également chargé d'organiser un service météorologique pour l'ensemble du territoire national ${ }^{52}$.

Figure 13

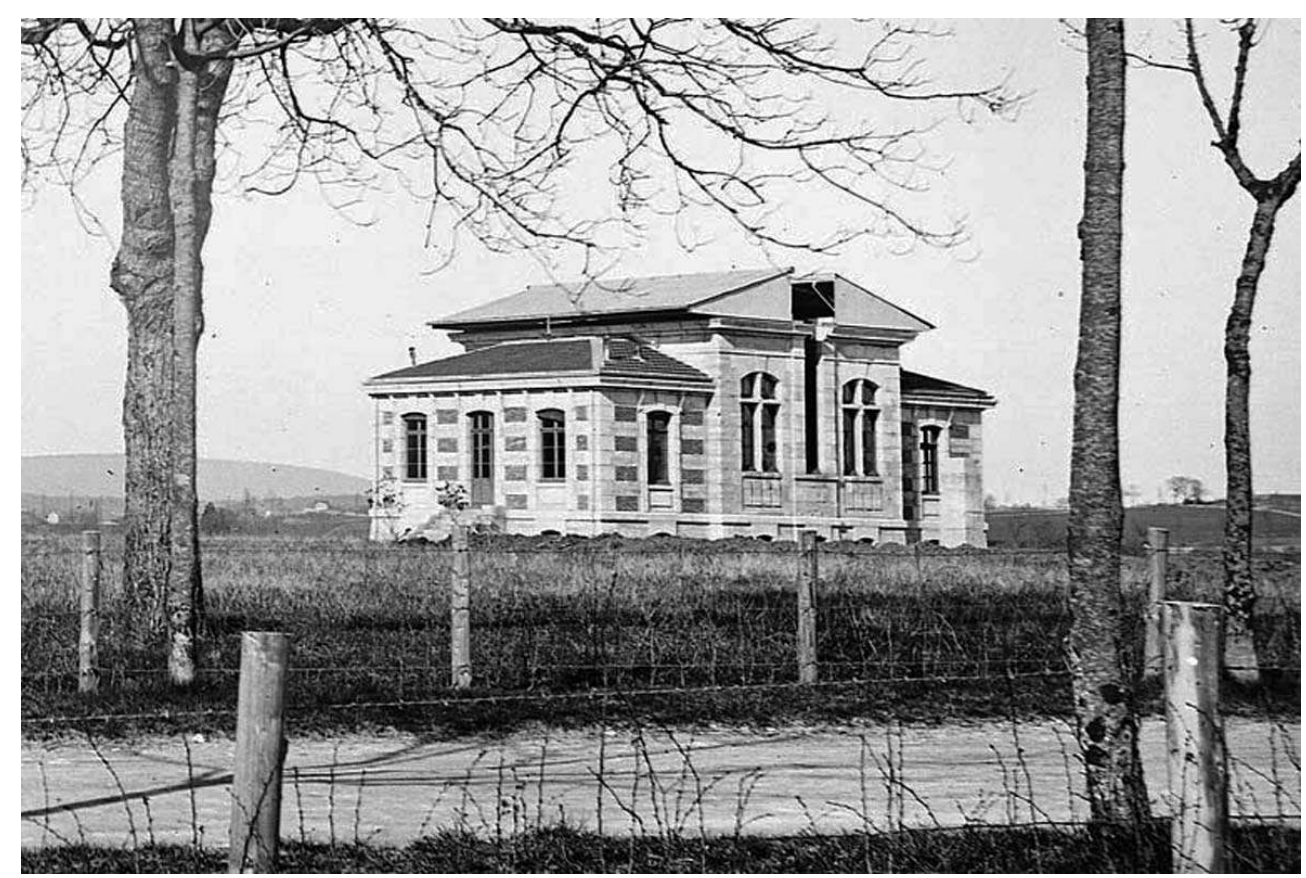

L'abri avec toit à deux longs pans du cercle méridien de l'observatoire de Besançon. Détail d'une photographie sur plaque de verre, années 1880. Archives de l'observatoire de Besançon.

Reproduction Jérôme Mongreville, 2007 @ Région Franche-Comté, Inventaire du patrimoine Observatoire des sciences de l'univers de Besançon.

Lorsque vingt ans après la livraison du cercle méridien par Gautier, Morize se préoccupa de commander un abri en vue de l'installation pérenne de l'instrument ${ }^{53}$, Gautier venait de mourir et en France aucun nouveau cercle méridien n'avait été installé depuis celui de Toulouse en 1890. Par contre, à Hambourg, ville où étaient installés les célèbres ateliers Repsold, l'observatoire venait de quitter son emplacement historique près des remparts de la ville ancienne pour s'installer à $20 \mathrm{~km}$ de là, dans un vaste site à la lisière du village de Bergedorf. Le nouvel observatoire allemand, dont la construction commença en 1906 et fut achevée en 1912, comprenait notamment un cercle méridien de Repsold équipé d'une lunette de $19 \mathrm{~cm}$ de diamètre abrité dans un bâtiment en bois équipé d'un toit ouvrant commandé à la maison Zeiss de Iéna ${ }^{54}$. Les principes de l'abri et de la fente d'ouverture différaient notablement de celui des cercles Eichens ou Gautier, notamment en ce qui concerne les matériaux et la conception architecturale. Les salles d'observation des cercles méridiens construits en France et à Alger à la fin du xixe siècle étaient en pierre, possédaient un toit mobile à deux longs pans et disposaient ou non de pièces annexes directement accolées de part et d'autre ${ }^{55}$ (fig. $\mathbf{n}^{\circ} \mathbf{1 3}$ ). Chez Zeiss, la salle d'observation, en bois et non en pierre, était recouverte d'un toit tonneau avec système d'aération et un escalier extérieur couvert la reliait à une "pièce-vestibule" située en contrebas de la salle ${ }^{56}$. C'est logiquement le nouveau modèle développé par Zeiss que Morize retint pour abriter de façon pérenne le cercle méridien Gautier de Rio de Janeiro - instrument dont le gabarit était très proche de celui du cercle méridien construit par Repsold pour Bergedorf (fig. $\mathbf{n}^{\circ} \mathbf{1 4}$ ). 
Figure 14

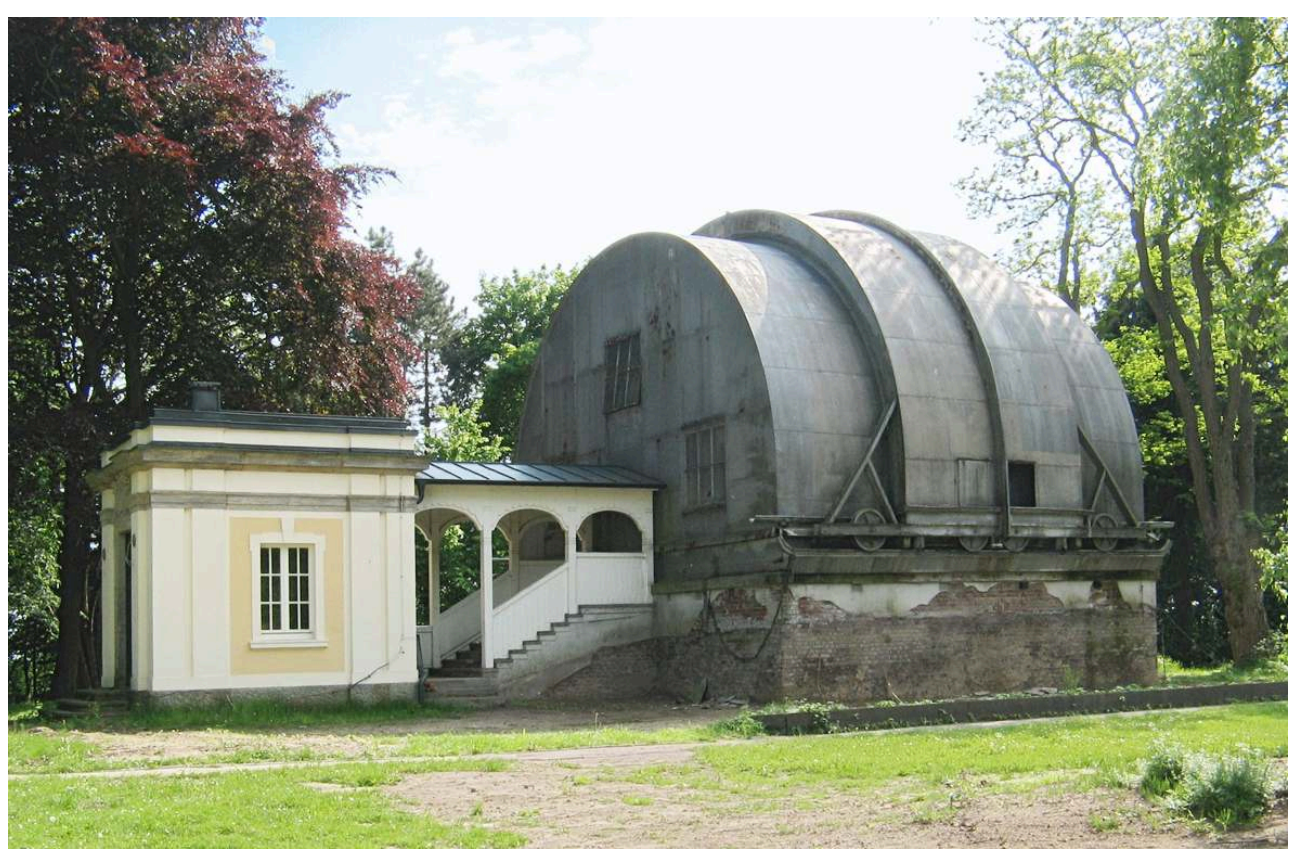

L'abri Zeiss à toit tonneau du cercle méridien Repsold de l'observatoire de Bergedorf (Hambourg Allemagne).

Vue depuis le nord-est. Photo. Marc Heller @ Marc Heller, 2004.

La construction de cet abri fut réalisée en 1915 par la maison João de Mattos Travassos Filho $^{57}$. Malheureusement, l'installation du toit ouvrant Zeiss fit apparaître des défauts de conception, notamment en termes d'étanchéité. L'eau de pluie pénétrant à l'intérieur de l'abri, allant même parfois jusqu'à tomber sur l'instrument lui-même, il fallut effectuer d'importantes réparations avant de procéder aux tests et aux essais sur le ciel et mettre en place un véritable service méridien. Selon un document envoyé par l'observatoire au ministre de l'Agriculture, de l'Industrie et du Commerce, les travaux de détermination de la position des étoiles en vue de l'amélioration du service de $l^{\prime}$ heure commencèrent le 30 mars $1928^{58}$. (fig. $\mathbf{n}^{\circ}$ 15) 
Figure 15

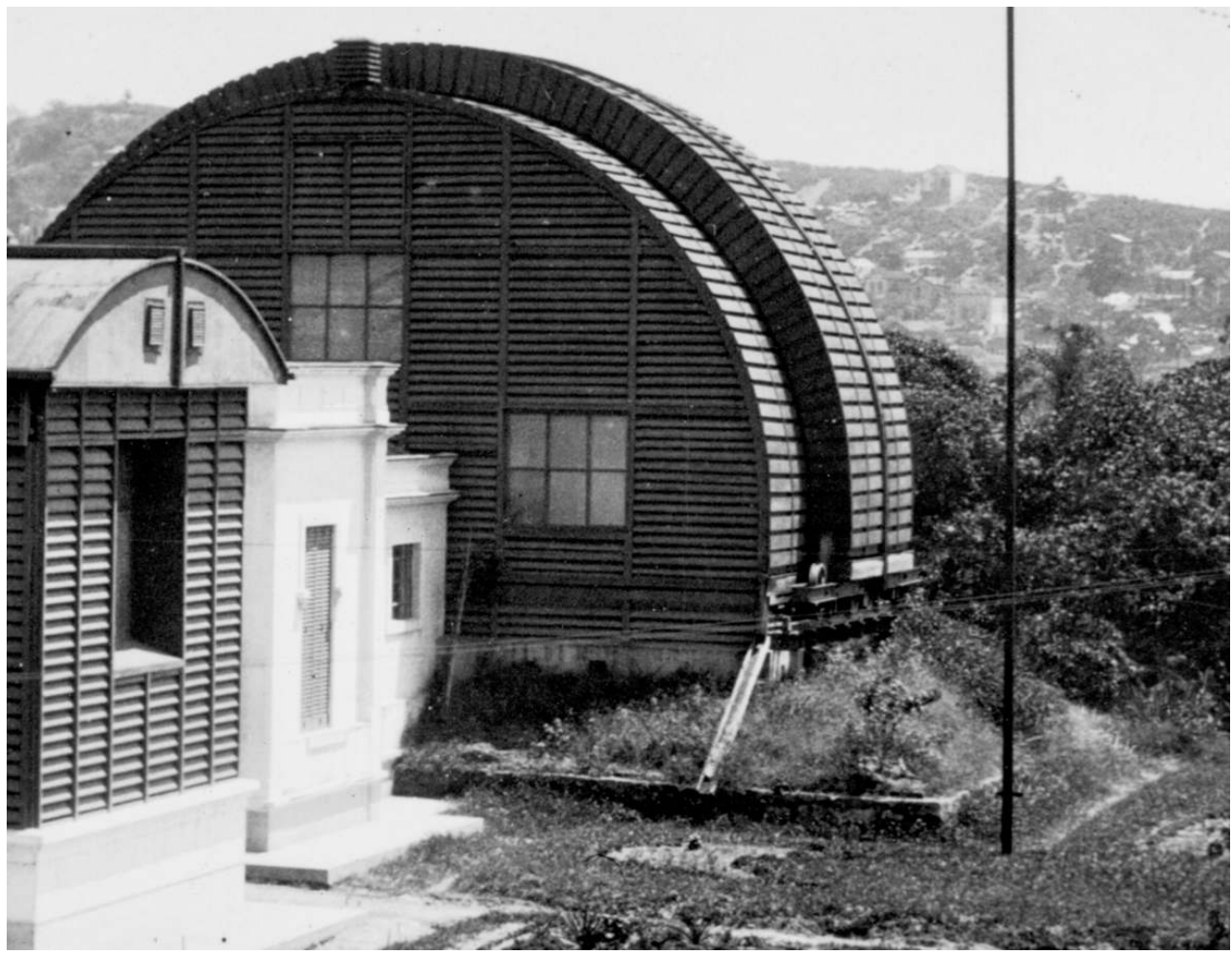

L'abri à toit tonneau destiné au cercle méridien Gautier de l'Observatoire national, colline de São Januário. Photographie vers 1928.

Archives du Museu de Astronomia e Ciências Afins, Rio de Janeiro, Brésil @ MAST.

Lors de l'installation de ce service, l'instant de passage des astres au méridien était repéré à l'aide d'une pendule synchronisée française de chez Leroy \& $\mathrm{Cie}^{59}$ et d'un chronographe imprimant nord-américain fabriqué par la maison nord-américaine Gaertner Precise Instrument Company of Chicago ${ }^{60}$. 
Figure 16

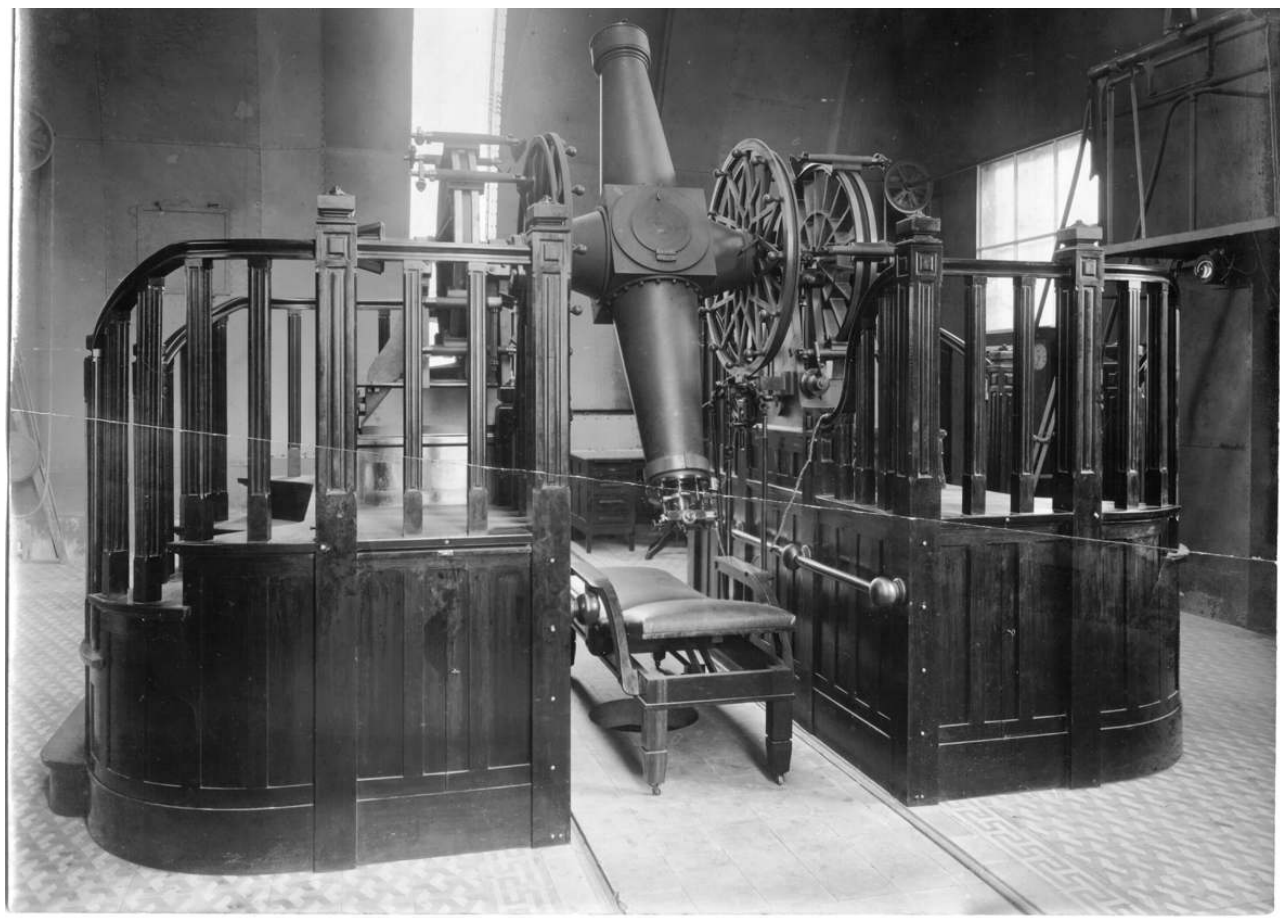

Le cercle méridien Gautier installé à l'Observatoire national, colline de São Januário. Photographie vers 1928.

Archives du Museu de Astronomia e Ciências Afins, Rio de Janeiro, Brésil @ MAST.

50 Les illustrations du cercle méridien les plus anciennes connues à ce jour correspondent à la période postérieure à l'installation sur la colline de São Januário, alors que l'instrument se trouve déjà à l'intérieur de l'abri Zeiss. (fig. $\mathbf{n}^{\circ}$ 16)

\section{Le site de São Cristovão hier et aujourd'hui}

Durant la période d'installation de l'Observatoire national à São Cristovão, l'acquisition de nouveaux instruments ainsi que la mise en fonction des instruments transférés du Morro do Castelo et de ceux nouvellement acquis furent ralentis par de nombreux obstacles institutionnels et financiers. Ainsi fallut-il des années pour que certains instruments soient réparés ou pour que d'autres soient livrés. Ceci eut évidemment pour conséquence l'absence de mise à niveau de l'instrumentation destinée aux travaux scientifiques. À ce premier handicap s'ajouta un manque criant de personnel - à la fois en nombre pour les tâches techniques et en qualification pour l'encadrement scientifique. Ainsi l'étude de la variation des latitudes que l'observatoire avait entreprise dut-elle être abandonnée faute de calculateurs ${ }^{61}$.

Ces deux facteurs - manque de personnel technique, sous-qualification des scientifiques - illustrent une caractéristique des premières années de la République brésilienne : l'absence d'institutionnalisation des activités de recherche. Cette dernière ne devint effective qu'à partir du moment où l'observatoire réussit à être doté des instruments nécessaires à ses travaux de recherche, c'est-à-dire durant la seconde moitié du xxe siècle. Chaque directeur s'efforça alors d'équiper l'observatoire des instruments les plus modernes - attitude héritée des directeurs de l'époque impériale qui avaient réussi à donner aux travaux entrepris à l'observatoire une dimension internationale. Mais, si les 
directeurs successifs furent bien conscients des impératifs tant institutionnels que financiers qu'imposait la pratique d'une astronomie moderne, ils rencontrèrent de nombreuses difficultés pour parvenir à leurs fins. Quoi qu'il en soit, un grand nombre d'instruments scientifiques - souvent de grande qualité - furent acquis pour l'accomplissement des différentes tâches de service et de recherche assignées à l'établissement.

Lorsque vers le milieu du xxe siècle, certaines des activités traditionnelles de l'observatoire national commencèrent à être abandonnées, la pendule Leroy du cercle méridien ainsi que quelques-uns des accessoires furent récupérés pour être utilisés à d'autres fins. Puis - selon le témoignage oral d'un ancien fonctionnaire de l'observatoire ${ }^{62}$ - en 1962 le cercle méridien fut entièrement démonté. L'année suivante, le revêtement intérieur de la pièce d'entrée du pavillon fut arraché en raison de la présence de termites. L'abandon du bâtiment ainsi que l'état d'oxydation avancé de ses parties métalliques et la grave détérioration des éléments en bois conduisirent entre 1980 et 1985 à sa destruction. Seules subsistèrent la partie en maçonnerie du vestibule et le soubassement de l'abri à toit tonneau ouvrant. Le passage couvert entre le vestibule et la salle d'observation fut condamné et l'état des parties maçonnées continua progressivement à se dégrader. (fig. $\mathrm{n}^{\circ} 17, \mathrm{n}^{\circ} 18$ )

Figure 17

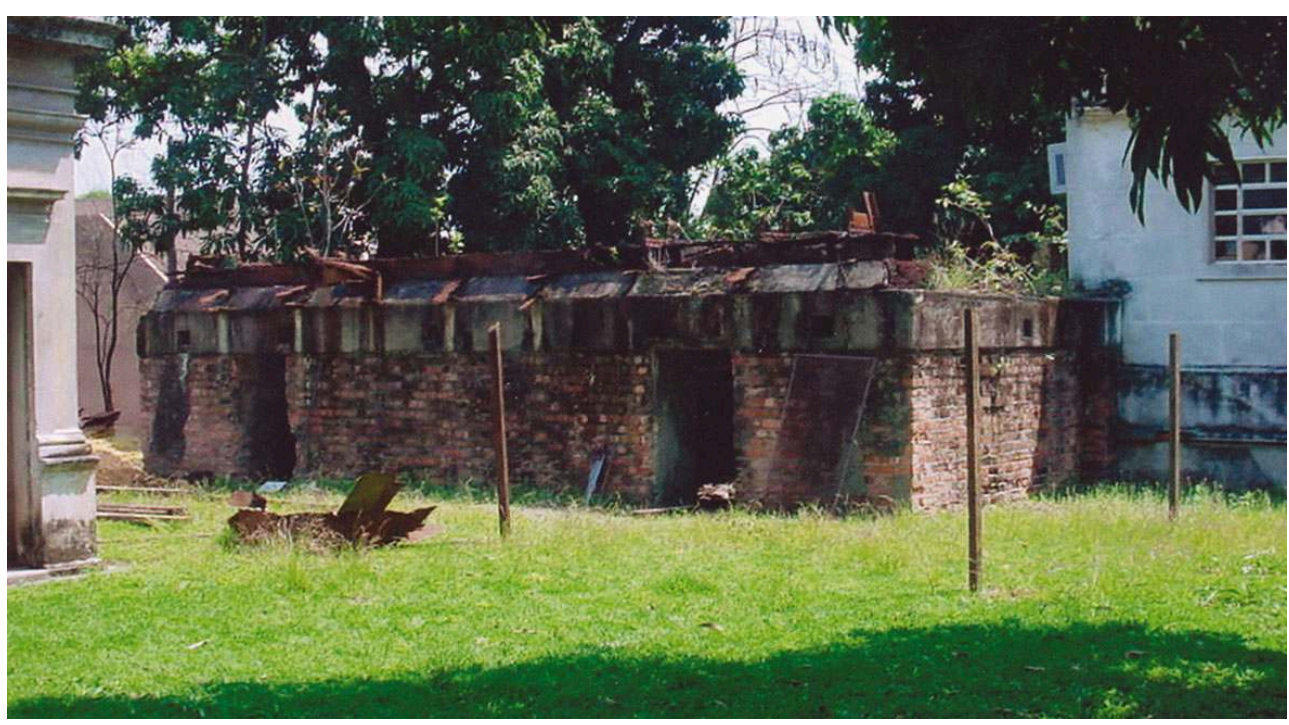

Massif en maçonnerie qui supportait le toit tonneau de l'abri du cercle méridien de l'observatoire de Rio de Janeiro, avant restauration. Le toit a totalement disparu, seuls subsistent les vestiges du plancher parqueté de la salle d'observation.

Archives du Museu de Astronomia e Ciências Afins, Rio de Janeiro, Brésil @ MAST. 
Figure 18

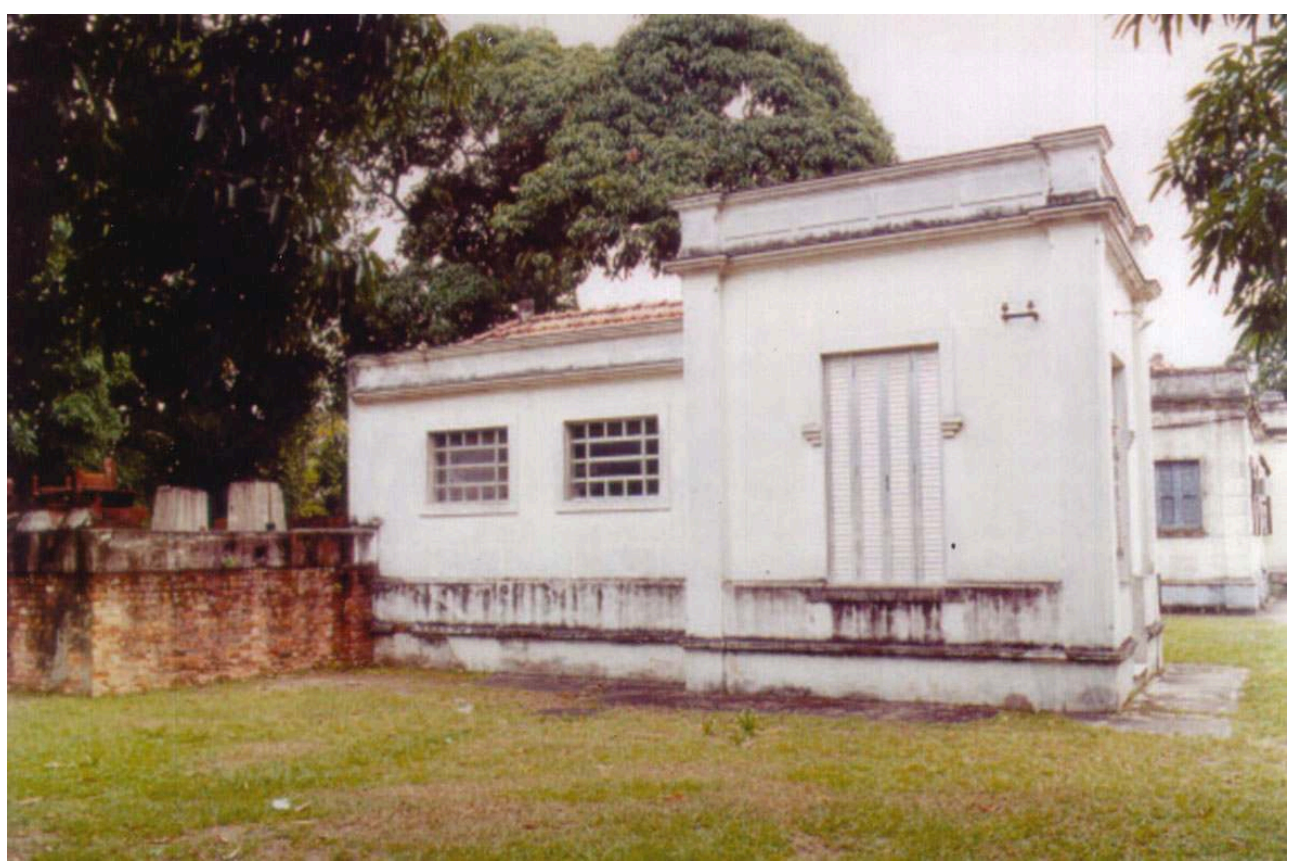

Bâtiment abritant la « pièce-vestibule ». Couloir d'accès à la salle d'observation et massif en maçonnerie qui supportait le toit tonneau de la salle d'observation méridienne, avant restauration. Archives du Museu de Astronomia e Ciências Afıns, Rio de Janeiro, Brésil @ MAST.

Lors de la création du Museu de Astronomia e Ciências Afins (MAST) en 1985, l'instrument était donc démonté et à l'abandon depuis plus de vingt ans et les fondations de la salle d'observation étaient menacées d'effondrement. La responsabilité de ces dernières fut attribuée au MAST, tandis que l'observatoire national conservait celle du vestibule. L'ensemble architectural, qui, comme on l'a vu, fut protégé au titre $\mathrm{du}$ patrimoine historique, comprenait naturellement les deux parties ainsi que le passage couvert permettant d'aller de l'un à l'autre. Quant à l'instrument démonté, il fut placé sous la responsabilité du MAST et fut protégé à la fois au titre de l'agence du patrimoine brésilienne, l'IPHAN, ${ }^{63}$ et de l'équivalent de cette agence dans l'État de Rio de Janeiro, l'INEPAC ${ }^{64}$. Ses différentes parties étaient stockées dans une pièce du rez-dechaussée du bâtiment qui abrite aujourd'hui le musée, à l'exception de certains éléments qui se trouvaient dispersés sur le campus.

Aujourd'hui, après l'importante opération de restauration décrite ci-dessous, le grand cercle méridien Gautier est présenté au public in situ dans un bâtiment reconstruit, tandis que les petits instruments et accessoires acquis au fil du temps par les astronomes de Rio et devenus obsolètes sont conservés dans les collections du MAST.

Quant à l'Observatoire national, implanté sur son site historique depuis près d'un siècle, il est installé dans des bâtiments nouveaux - inaugurés en 1985 -, et il continue à être un centre de recherche très actif. 


\section{Des fragments au tout : la restauration du cercle méridien Gautier de l'observatoire de Rio de Janeiro}

\section{Réflexions préalables à la restauration du cercle méridien Gautier et de son abri}

La question de la restauration de cet instrument prestigieux devint à partir de 1997 l'une des grandes questions débattues dans le département de muséologie du MAST. Qu'il s'agisse d'instruments scientifiques ou non, il convient en effet avant toute restauration de se poser un certain nombre de questions, la première d'entre elles étant : compte tenu de son coût en moyens financiers et en temps, l'opération en vautelle la peine?

En 1993, Brenni et Miniati ${ }^{65}$ suggérèrent certains critères potentiels de décision tels que rareté, ancienneté, complexité et origine de l'objet. Ils soulignèrent cependant qu'un même instrument placé dans des contextes différents peut être porteur de sens différents. Ainsi, une machine électrostatique de série extrêmement commune à la fin du xixe siècle ne devrait-elle pas être restaurée, sauf si elle appartient à une collection homogène et complète d'appareils de la même époque et qu'en son absence l'intérêt de la collection en question serait amoindri.

En ce qui concerne le cercle méridien Gautier, les principaux arguments avancés en faveur de sa restauration furent sa rareté - une douzaine d'exemplaires dans le monde (voir tableau ci-dessus) -, la complexité de son principe, l'intérêt historique de sa présence au Brésil, et la réputation de son constructeur - Gautier fut à la fin du xixe siècle l'un des principaux fabricants mondiaux de grands instruments astronomiques.

Par ailleurs, de tous les grands instruments du campus abrités dans des bâtiments appartenant à l'ensemble architectural historique de l'observatoire, il était le seul à être démonté. De ce fait, non seulement l'instrument était menacé à terme de disparaître définitivement, mais il était le seul grand instrument non en place dans son abri spécifique. Il convient de souligner à ce propos que la protection au titre de patrimoine historique de l'ensemble architectural témoigne de l'intérêt exceptionnel de ce dernier : non seulement les grands instruments astronomiques historiques abrités dans les bâtiments protégés sont encore implantés dans leur site d'origine, mais, compte tenu de l'histoire de l'institution, ils n'ont pas subi de transformations qui peu ou prou auraient pu les dénaturer.

61 Le fait d'appartenir à un ensemble architectural protégé a d'ailleurs déterminé les choix opérés concernant la restauration de l'abri du cercle méridien. Compte tenu du parti pris concernant la restauration de l'instrument - non pas un retour à ses fonctionnalités d'origine, mais une présentation au public permettant sa compréhension au sein d'un espace muséal dédié -, il fut décidé de reconstruire l'abri à toit tonneau à l'identique du point de vue des matériaux, du volume et de l'apparence afin qu'il s'intègre harmonieusement dans l'ensemble architectural existant. Dans le but de privilégier non les fonctionnalités de l'instrument et de son abri (possibilité d'observations astronomiques diurnes ou nocturnes), mais leur dimension «potentiel de communication ", l'espace correspondant à l'ancienne salle d'observation abrite donc désormais un espace muséal dédié à cet instrument scientifique devenu objet 
patrimonial mais (ré)installé dans son emplacement d'origine historique. (fig. $\mathbf{n}^{\circ} \mathbf{1 9}$, $\mathbf{n}^{\circ}$ 20)

\section{Figure 19}

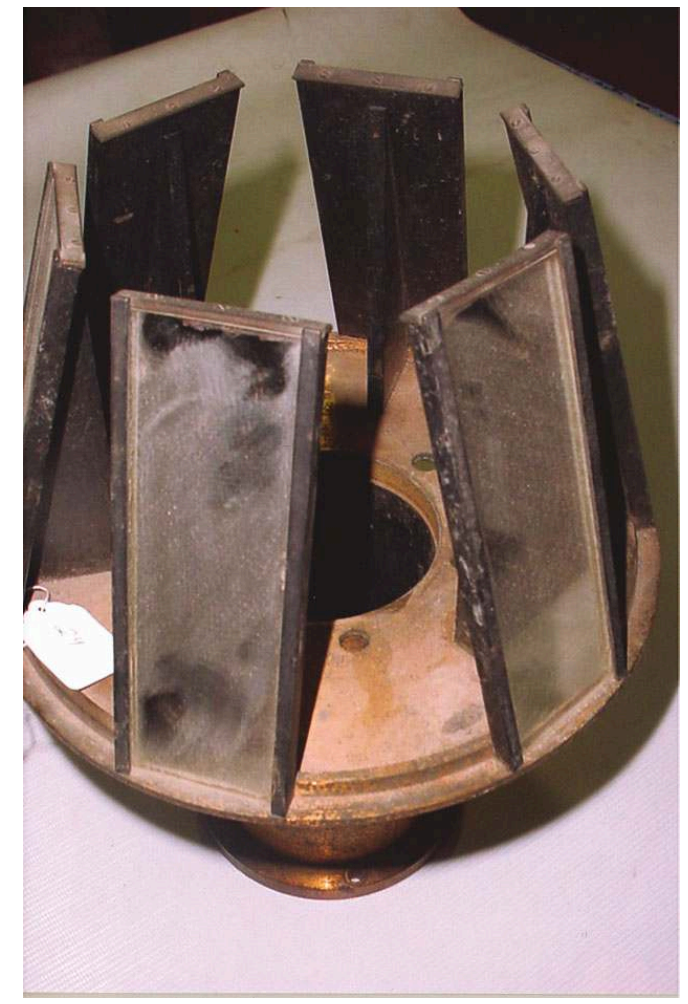

Dispositif avec miroirs de renvoi pour l'éclairage du cercle de lecture, avant restauration. Archives du Museu de Astronomia e Ciências Afins, Rio de Janeiro, Brésil (c) MAST. 


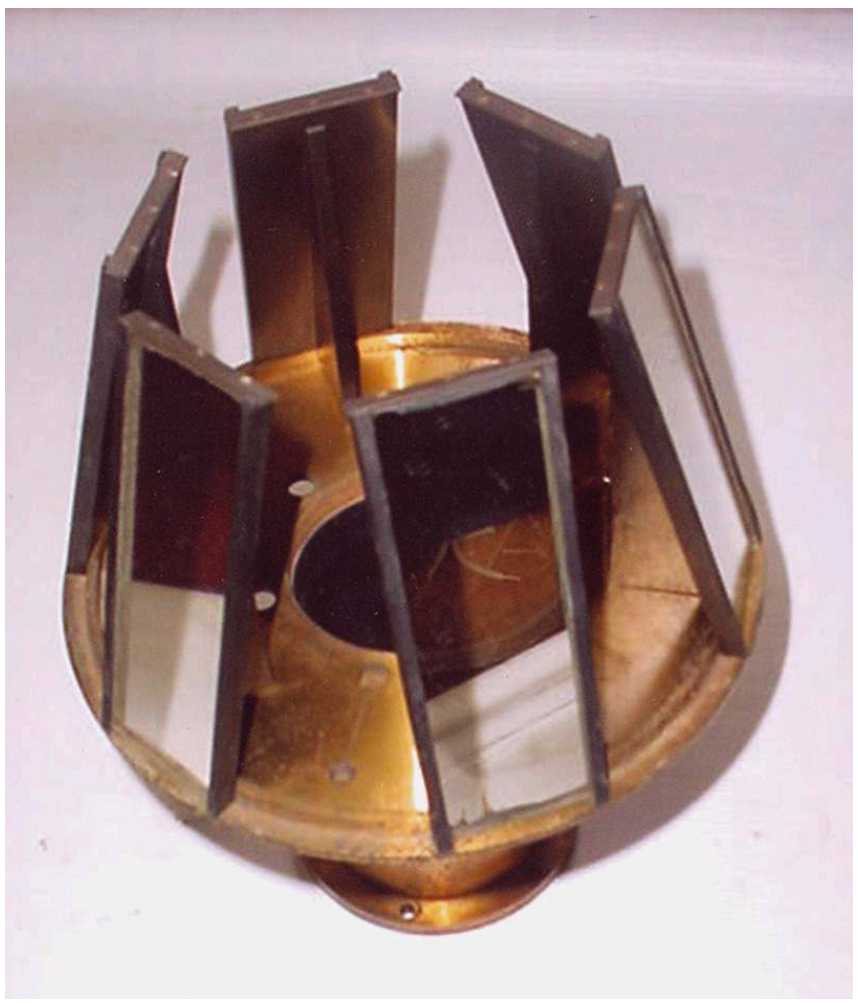

Dispositif avec miroirs de renvoi pour l'éclairage du cercle de lecture, après restauration. Archives du Museu de Astronomia e Ciências Afıns, Rio de Janeiro, Brésil @ MAST.

\section{Opérations préalables à la restauration}

Une fois qu'il fut décidé que cercle méridien méritait d'être restauré, l'étape suivante consista, comme pour les objets d'art, à faire un diagnostic approfondi de son état de conservation afin d'être en mesure de programmer les interventions à réaliser. Il est intéressant de signaler que, à ce moment particulièrement critique de l'opération, l'équipe n'eut pas à considérer la restauration d'un objet unique, mais plutôt celle d'un grand nombre de pièces, qui de plus étaient dispersées en divers lieux. Pire, personne ne savait si toutes les parties de l'instrument avaient été conservées ou du moins si les parties indispensables pour remonter l'instrument existaient encore. Aussi, avant même de discuter de la manière de restaurer l'instrument, un recensement des observatoires et des musées des sciences et des techniques fut réalisé (France, Allemagne, États-Unis, Australie, Algérie) afin d'identifier ceux qui possédaient des cercles méridiens du même fabricant, en vue de mieux identifier les parties d'instrument conservées dans la réserve technique du MAST.

Les contacts pris furent fructueux. Les observatoires d'Alger, de Besançon et de Toulouse possédaient des cercles méridiens Gautier très semblables à celui de l'Observatoire national. Installés respectivement en 1888, 1885 et 1890, ces instruments étaient munis d'objectifs de même diamètre $(19 \mathrm{~cm})$ que celui de Rio et de focales très voisines ( $240 \mathrm{~cm}$ pour Alger, $237 \mathrm{~cm}$ pour Besançon et $230 \mathrm{~cm}$ pour Toulouse).

Grâce aux nombreuses photographies numériques envoyées par ces observatoires, il fut possible à la fois de conclure à la faisabilité de la restauration du cercle méridien de Rio 
et d'identifier les quelques pièces manquantes ${ }^{66}$. Une nouvelle étape de l'opération fut ensuite entamée, à savoir un véritable travail d'enquête, mené sur tout le campus occupé par l'observatoire et par le MAST, à la recherche des pièces manquantes. Tous les tiroirs, placards et salles du musée furent à nouveau explorés et de nombreux contacts furent établis avec des personnels de l'observatoire, ce qui permit d'identifier quelques pièces appartenant à l'instrument et de les ajouter à celles déjà rassemblées par ailleurs. Parmi les éléments importants récoltés à cette occasion, il faut mentionner notamment: deux axes en laiton avec poignée à l'une des extrémités provenant du système de blocage ; deux barres en fer avec système de fixation servant à maintenir les contrepoids ; deux plaques carrées en laiton ; un ensemble de vis en laiton.

Après avoir rassemblé toutes les informations, puis organisé et classé les différents éléments retrouvés, on évalua pour chacun d'eux la nécessité d'une restauration. Seules quatre pièces ne nécessitaient aucune intervention: les deux cônes du système d'éclairage à miroirs, le micromètre de Gautier et le micromètre de Bouty. Quant aux autres pièces, elles étaient dans un état assez critique : vernis original détérioré, pièces peintes d'une couleur différente de celle d'origine et peinture en mauvais état, vastes surfaces métalliques sévèrement corrodées avec dommages mécaniques. (fig. $\mathbf{n}^{\circ} \mathbf{2 1}$, $n^{\circ}$ 22)

Figure 21

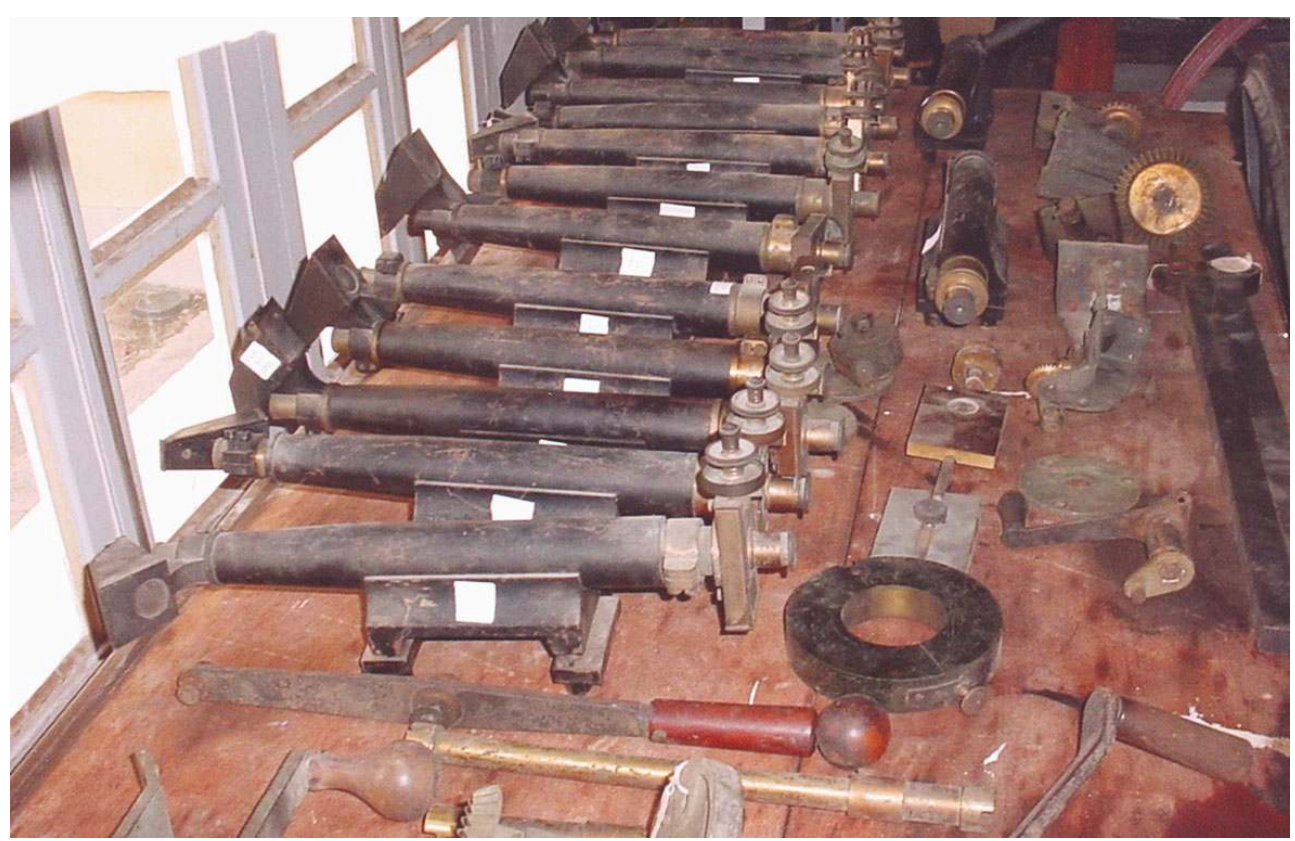

Microscopes de lecture des cercles gradués munis de leur micromètre, avant restauration. Archives du Museu de Astronomia e Ciências Afins, Rio de Janeiro, Brésil @ MAST. 


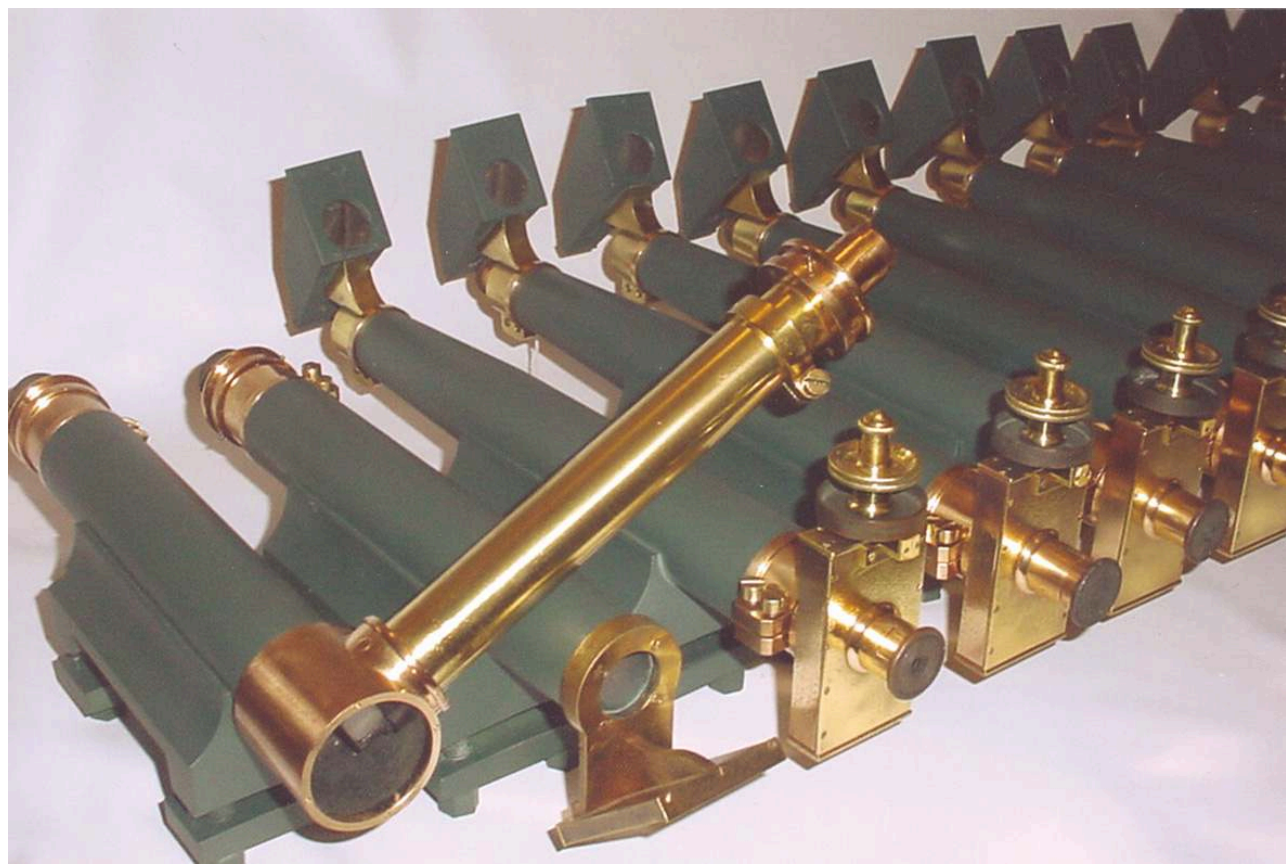

Microscopes de lecture des cercles gradués munis de leur micromètre, après restauration. Archives du Museu de Astronomia e Ciências Afins, Rio de Janeiro, Brésil @ MAST.

\section{Les étapes de la restauration-reconstruction de l'instrument}

Le micromètre d'origine du cercle méridien, fabriqué par le même constructeur que l'instrument, est un micromètre impersonnel de Gautier. Cependant, lorsque ce micromètre fut examiné selon les règles déontologiques s'appliquant au patrimoine matériel, il apparut que certaines de ses parties en avaient été retirées afin d'être adaptées en 1923 au micromètre Bouty ${ }^{67}$. Il fut alors décidé d'installer sur le cercle méridien non le micromètre Gautier d'origine, mais le micromètre Bouty, étant donné que c'est ce nouveau micromètre qui fut utilisé lorsque les observations commencèrent, en 1928. Quant au micromètre Gautier d'origine, il fut décidé de le présenter dans une vitrine à l'intérieur du pavillon méridien avec les autres pièces faisant l'objet d'une présentation muséographique. Le micromètre Gautier étant en bon état, il n'eut pas à être restauré.

67 L'examen du micromètre Bouty fit apparaître que sa base circulaire en laiton provenait du micromètre Gautier d'origine. L'inscription gravée sur cette pièce indique Gautier 1893 Bouty 1923, ce qui corrobore l'hypothèse d'une adaptation réalisée par Bouty. Après nettoyage, ce micromètre fut fixé sur la partie oculaire du tube optique à l'aide des vis d'origine. Suite à l'opération de diagnostic menée précédemment, la phase «hygiénisation » de toutes les pièces du cercle méridien fut entreprise sous la forme d'un nettoyage mécanique des surfaces. Puis fut discutée la philosophie générale de la restauration à entreprendre.

Comme on l'a souligné précédemment, il existe depuis longtemps deux courants de pensée en vigueur dans le domaine des instruments scientifiques. L'un, préconisé par les collectionneurs, les techniciens et les physiciens, est favorable à une restauration en profondeur de l'objet, l'objectif primordial étant la remise en fonction de l'instrument. 
Il est intéressant à ce sujet de rappeler que, selon de nombreuses études, les objets scientifiques et techniques sont considérés comme différant des autres objets culturels en ce qu'ils présentent une dimension «fonctionnement » qui devrait prévaloir sur les autres dans le cas d'une restauration. L'autre courant de pensée - soutenu par les restaurateurs d'art et les historiens - privilégie quant à lui une restauration légère qui ne remplace aucune pièce et ne répare pas l'objet. Miniati et Brenni ont quant à eux proposé une voie médiane moins radicale :

Dans de nombreux cas, il est nécessaire de remplacer des parties disparues : si on connaît avec certitude l'état d'origine de l'instrument, il est alors possible de procéder à la reconstruction de la pièce ou des pièces manquante(s). Nous ne partageons pas le fétichisme pour les matériaux anciens de ceux qui, sous prétexte de ne pas toucher à la poussière du temps - elle-même souvent simple saleté-, se contentent de conserver une relique de peu d'utilité.

70 Naturellement, chaque intervention devra être réversible: les parties refaites seront décrites avec précision, ce descriptif sera reporté sur une fiche spéciale de restauration et, afin d'éviter toute erreur et toute confusion, on pourra placer sur les pièces refaites une petite marque destinée à distinguer les parties récentes des parties d'origine ${ }^{68}$.

71 Si à l'époque où ce texte fut écrit on considérait encore comme réversibles certaines actions effectuées à titre conservatoire, aujourd'hui toute intervention sur un objet culturel est considérée comme irréversible - y compris le simple nettoyage à l'aide d'une brosse ou d'un pinceau ${ }^{69}$. Aucune action ne serait donc réversible au sens strict. En conséquence, on utilise en lieu et place du concept d'irréversibilité celui d'intervention minimale nécessaire à la réalisation de l'objectif visé par l'intervention sur l'objet. En l'occurrence il s'agit essentiellement du potentiel de communication de l'objet.

72 D'autres auteurs ont aussi abordé la question du fonctionnement d'instruments appartenant à des collections muséologiques, dont Mohen ${ }^{70}$ - qui associe ces collections soit aux musées techniques soit aux musées de la musique. D’après cet auteur, ces types d'institutions seraient confrontés à la tentation de restituer la fonction initiale de leurs objets - par exemple faire sonner l'heure à une horloge ou jouer d'un violon lors d'un concert. Prenant l'exemple d'instruments de musique médiévaux dans un concert de musique ancienne, Mohen souligne qu'il est illusoire de penser recréer les conditions authentiques dans lesquelles ces instruments étaient utilisés parce que les instruments eux-mêmes ont changé à la suite des altérations physiques qu'ils ont subies au cours des ans - tout comme les interprètes et le public, qui eux aussi sont actuels. Le passé ne peut jamais être recréé.

73 En ce qui concerne le cercle méridien Gautier du MAST, le principe qui orienta sa restauration fut de ne pas l'utiliser à des fins didactiques d'expérimentation, donc de ne pas lui restituer sa fonction d'observation des astres. Il fut décidé de le considérer comme un élément muséologique à visée didactique au sein d'un nouvel espace d'exposition qui ne serait pas utilisable pour des démonstrations pratiques. En conséquence, les interventions qui auraient été justifiées en vue de rétablir la fonctionnalité de l'instrument ne furent pas réalisées. (fig. $\mathbf{n}^{\circ} 23, \mathbf{n}^{\circ} 24$ ) 
Figure 23

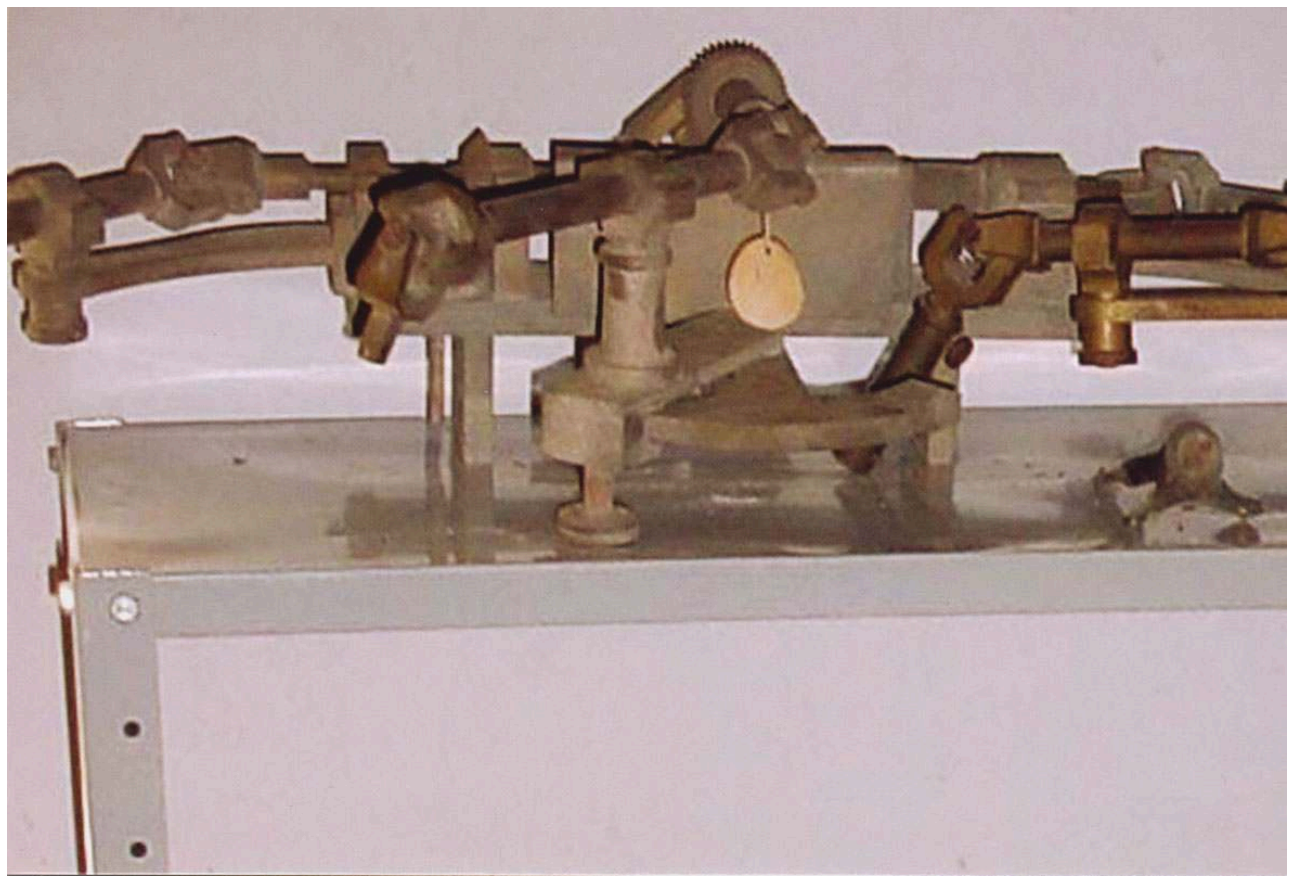

Dispositif pour le réglage fin de l'axe de visée de la lunette dans le plan méridien, avant restauration. Archives du Museu de Astronomia e Ciências Afins, Rio de Janeiro, Brésil @ MAST.

\section{Figure 24}

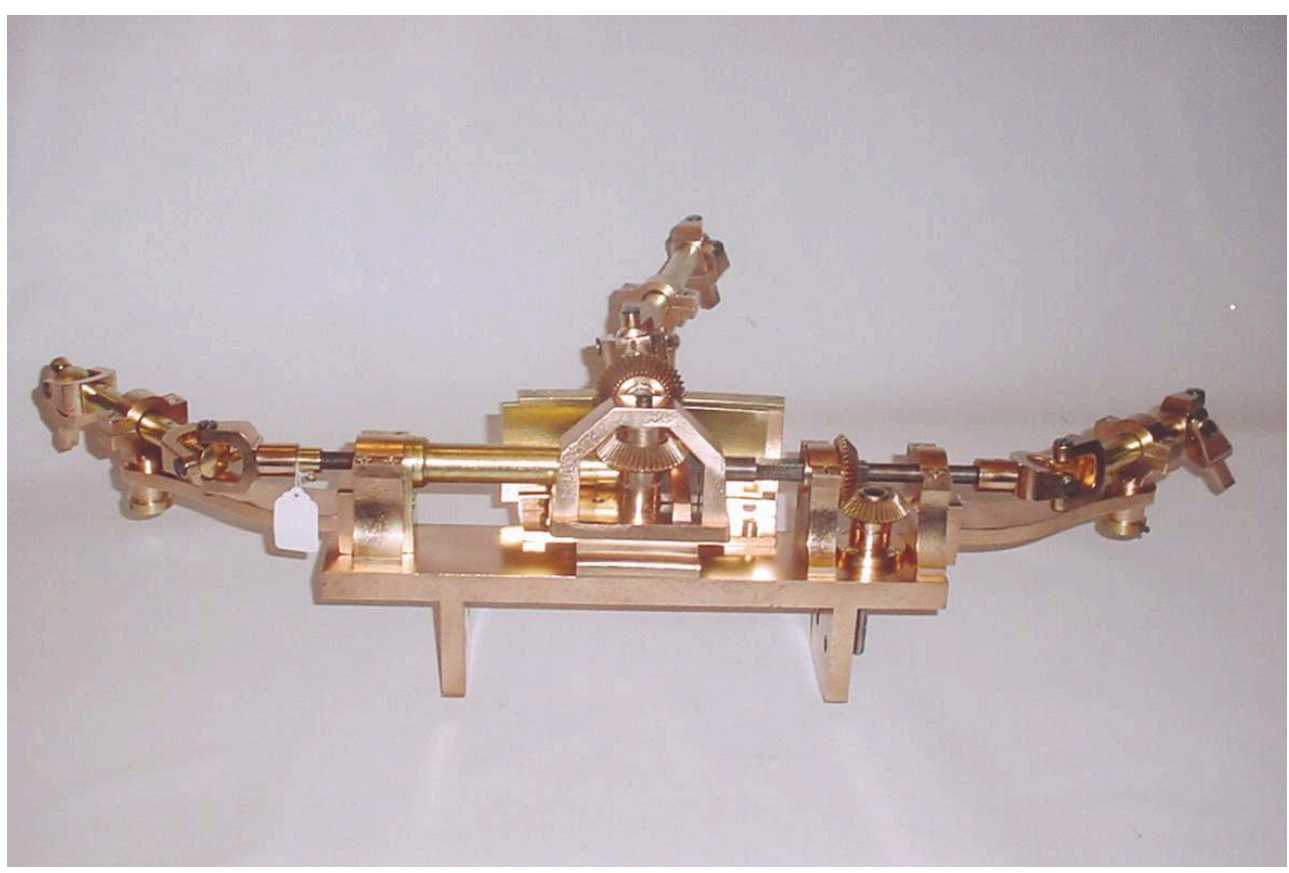

Dispositif pour le réglage fin de l'axe de visée de la lunette dans le plan méridien, après restauration. Archives du Museu de Astronomia e Ciências Afıns, Rio de Janeiro, Brésil @ MAST.

Dans un instrument de grande dimension tel que le cercle méridien, le nombre de vis est considérable et l'une des premières choses à discuter est la question du remplacement des vis perdues. Il est très tentant de les remplacer par des vis neuves 
même si cela nécessite de repercer les trous correspondants, cependant les altérations induites peuvent rendre l'opération éthiquement inacceptable. Wheatley ${ }^{71}$ recommande en cas de remplacement de vis d'utiliser des vis identiques aux originales. Dans le cas présent, seules quelques-unes durent être remplacées car la plupart de celles qui manquaient n'affectaient ni la structure ni la stabilité de l'instrument. Les vis manquantes véritablement nécessaires furent remplacées par des répliques des vis originales, répliques qui furent replacées dans les trous d'origine, simplement nettoyés. Aucun trou nouveau ne fut donc percé.

En ce qui concerne la troisième phase du processus, la phase de démontage de l'instrument à restaurer, il se trouve comme on l'a déjà dit que l'instrument avait été démonté dans les années 1960. Cette situation était d'autant plus regrettable que les règles de conservation actuellement en vigueur ne furent évidemment pas appliquées lors du démontage et que ne fut conservée aucune trace écrite de l'opération - qui de ce fait n'est donc pas documentée. Or, comme le souligne Keene, lors du démontage d'un instrument la question des vis est cruciale ${ }^{72}$ :

Même l'opération apparemment innocente de démontage d'un instrument en vue de procéder à son nettoyage et à son remontage peut provoquer des dommages. Par exemple, lorsqu'ils retirent des vis, beaucoup de gens ne se rendent pas compte que dans les instruments scientifiques chaque vis est fabriquée sur mesure pour un trou spécifique.[...] Il est indispensable de noter la position de chacune d'elles au moment du démontage. Les fentes sur les têtes des vis ayant souvent une forme spécifique, un conservateur habile et averti fabriquera un tournevis exactement adapté à cette forme, sinon les têtes des vis risquent d'être endommagées, comme c'est fréquemment le cas, et la surface de l'instrument abîmée.

Ainsi, faute de connaître la position de toutes les vis du cercle méridien, le remontage de celui-ci après restauration de ses différentes parties risquait d'aboutir à un résultat différent de l'original. Malgré ce handicap, il y eut consensus au sein de l'équipe en charge de la décision pour mener à bien l'opération de restauration. On présente cidessous de façon succincte les interventions réalisées sur un certain nombre des pièces qui furent restaurées.

Les procédures de restauration utilisées furent semblables à celles déjà mises en œuvre pour d'autres instruments appartenant aux collections du MAST ${ }^{73}$. Lorsque les pièces à restaurer possédaient encore des traces de vernis, elles furent tout d'abord nettoyées avec une solution de chlorure de méthylène. Ensuite, elles furent lavées à grande eau afin d'éliminer toute trace de la solution, puis séchées. La rouille des parties oxydées fut supprimée en utilisant des procédés mécaniques tels que pâtes à polir et papier de verre abrasif très fin. Au cours du processus final, on utilisa des papiers à grain 320 et 400. Par contre les papiers à grain plus fin (600 ou 720) ne furent pas utilisés afin de ne pas obtenir des surfaces métalliques trop brillantes par rapport à l'aspect du métal de l'instrument d'origine. Tout au long du processus mécanique, la surface des parties métalliques fut régulièrement nettoyée avec du coton afin de retirer l'huile mêlée aux produits d'oxydation en suspension. Enfin, lorsque la corrosion était ponctuelle en profondeur ${ }^{74}$ ou très localisée, on fit usage d'un scalpel dans ces parties spécifiques, ce qui évita le traitement de toute la surface de la pièce.

79 Les pièces produites à l'aide d'un tour et portant des traces de la fabrication furent traitées au tour pour éliminer les couches de corrosion, de façon à rétablir les cercles caractéristiques de cette sorte de fabrication. 
80 Après l'élimination des produits de corrosion, les pièces sans vernis furent dégraissées au trichloréthylène, puis recouvertes d'une couche de cire microcristalline. Les pièces avec vernis furent également dégraissées, puis immédiatement recouvertes à l'aide d'un aérographe d'une nouvelle couche de vernis.

81 Le choix de la peinture de protection des parties peintes à l'origine fut décidé à partir de l'identification dans certaines parties de l'objet de parties qui une fois démontées permettaient d'accéder à la peinture d'origine. Les parties extérieures furent recouvertes d'une peinture synthétique brillante composée de trois coloris de marque Coralmur (38\% de vert, code 9159, $38 \%$ de gris, code 9152, et $24 \%$ de bleu, code 9295). Pour certaines parties intérieures qui à l'origine étaient peintes en noir on utilisa une peinture noire mate (corps de la lunette, cube central et couvercle de l'objectif). (fig. $\left.n^{\circ} 25, n^{\circ} 26\right)$

Figure 25

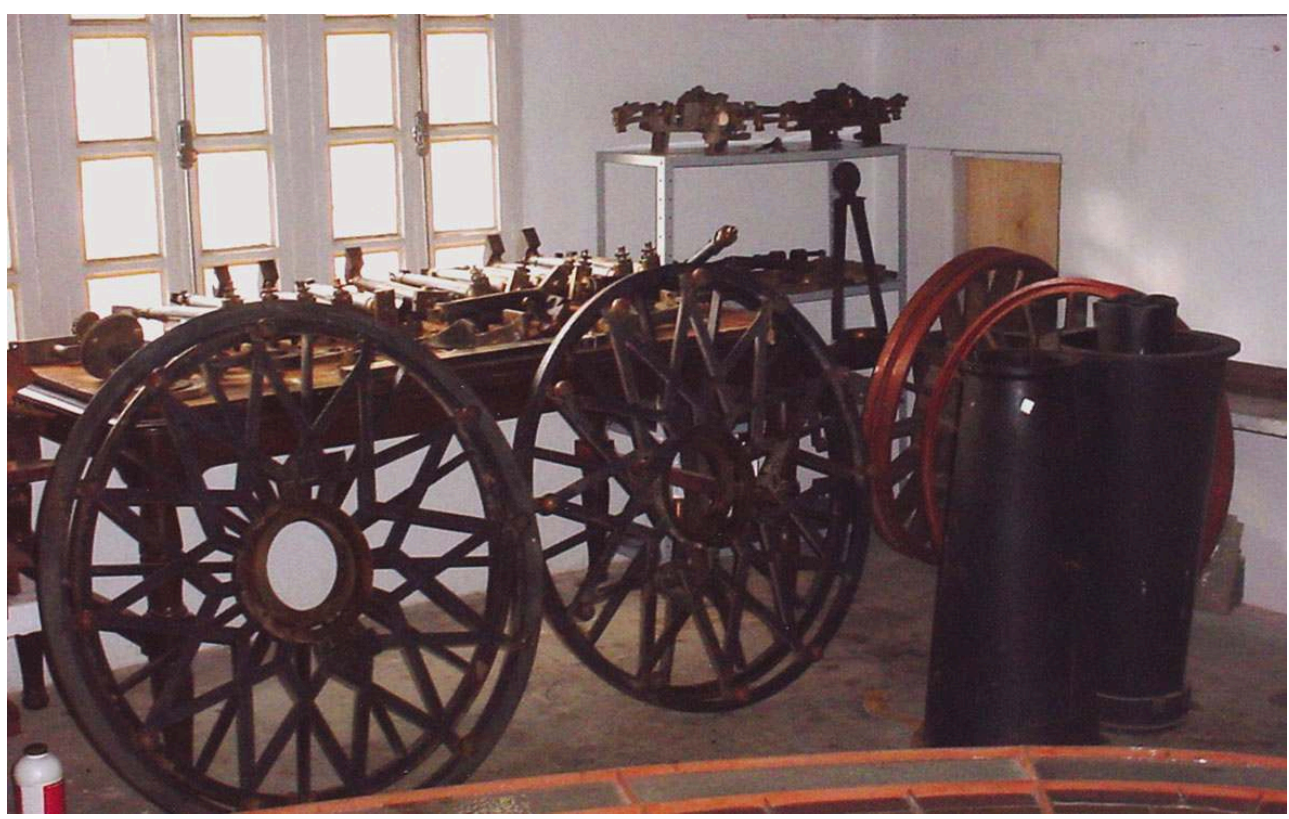

Différentes pièces du cercle méridien Gautier démonté, avant restauration. Archives du Museu de Astronomia e Ciências Afins, Rio de Janeiro, Brésil @ MAST. 
Figure 26

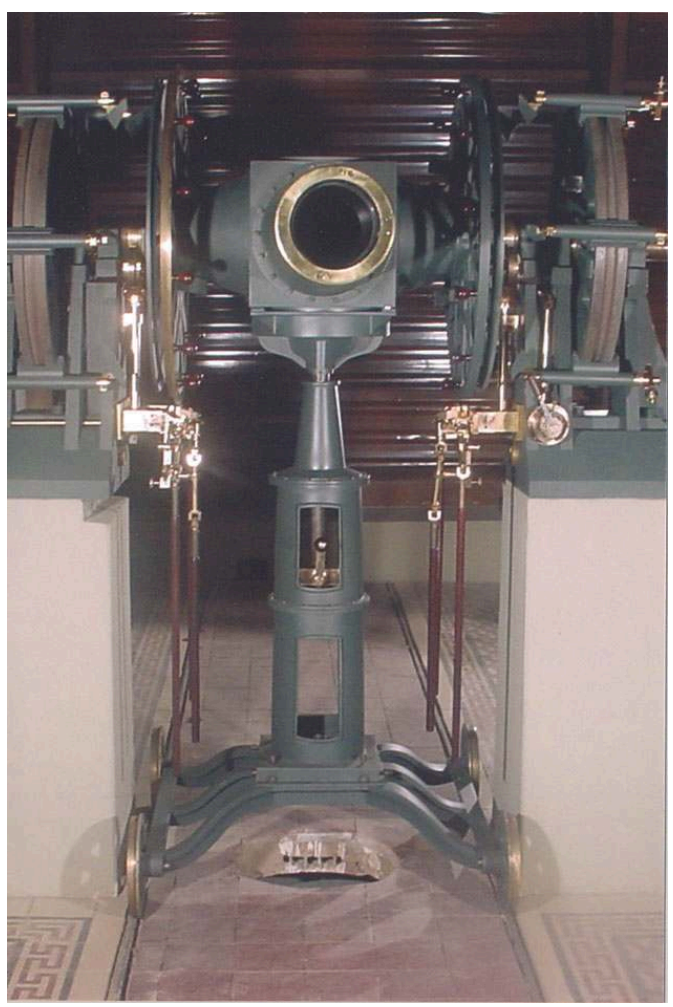

Le cercle méridien Gautier restauré et installé sur ses piliers dans l'abri reconstruit. Archives du Museu de Astronomia e Ciências Afins, Rio de Janeiro, Brésil @ C MAST.

82 Les travaux les plus complexes menés à ce jour au sein du MAST furent sans conteste la restauration du cercle méridien de Gautier et la rénovation de son abri. Mené par une équipe multidisciplinaire, ce projet s'appuya sur un corpus de recherches historiques relatif à l'instrument et son abri. L'opération, qui se déroula sur trois ans, fut accompagnée d'une campagne photographique exhaustive de chaque étape du travail, comprenant le diagnostic relatif à l'état de conservation de l'instrument, sa restauration, la rénovation du pavillon, la réinstallation de l'instrument dans son emplacement d'origine, ainsi que la muséalisation de l'espace, ce qui permet de fournir au public des informations sur l'opération de restauration elle-même. (fig. $\mathbf{n}^{\circ} \mathbf{2 7}$, $\mathbf{n}^{\circ}$ 28) 
Figure 27

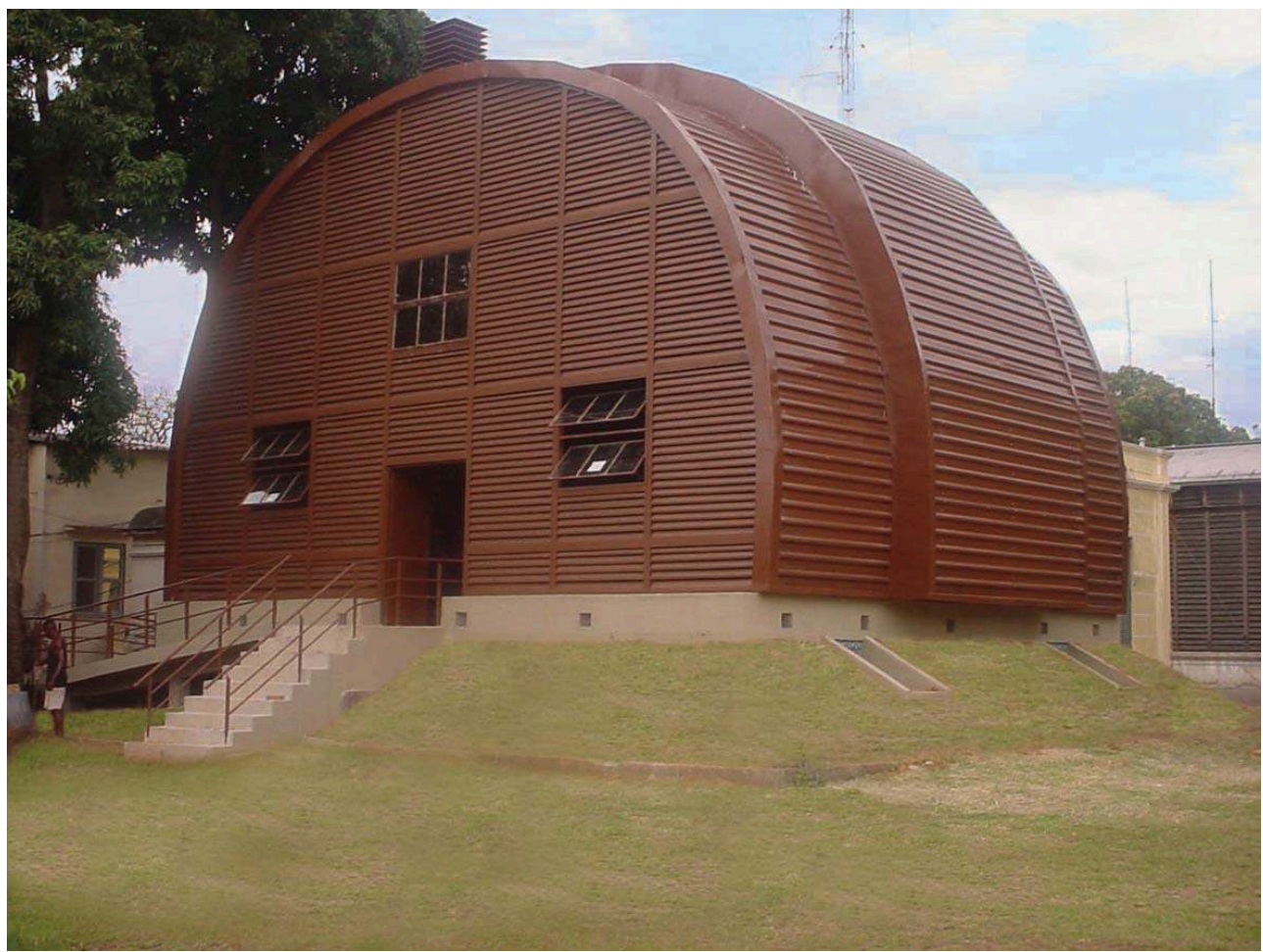

Le nouveau toit tonneau, non fonctionnel, de la salle d'observation méridienne.

Archives du Museu de Astronomia e Ciências Afins, Rio de Janeiro, Brésil @ C MAST. 
Figure 28

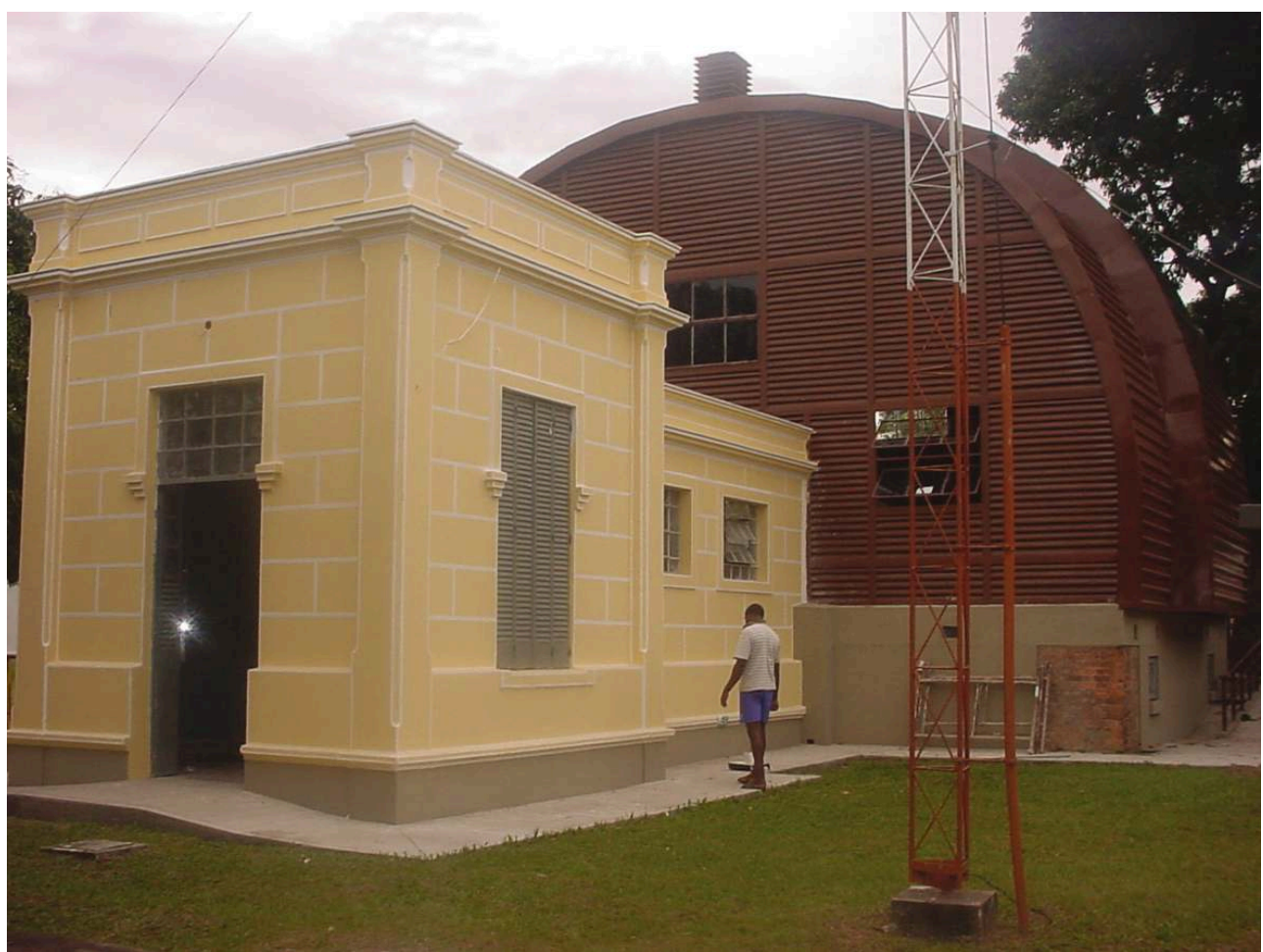

L'abri du cercle méridien après la restauration du bâtiment abritant « pièce-vestibule » et le couloir d'accès à la salle d'observation et après la construction du nouveau toit tonneau, non fonctionnel, pour la salle d'observation méridienne.

Archives du Museu de Astronomia e Ciências Afins, Rio de Janeiro, Brésil @ MAST.

\section{Considérations finales}

À l'exception de quelques grands instruments astronomiques dus aux frères Brunner ${ }^{75}$, la plupart des instruments fixes construits dans les observatoires français durant la période 1870-1910 provinrent tout d'abord des ateliers d'Eichens, puis de ceux de son successeur Gautier. Si Eichens construisit vers 1875 un cercle méridien pour l'observatoire de Lima (fig. $\mathbf{n}^{\circ}$ 29), Gautier quant à lui créa une véritable sphère d'influence française en matière d'instruments astronomiques en livrant de grands instruments dans des observatoires d'Europe (Autriche, Espagne, Grèce, Pays-Bas, Roumanie, Vatican), d'Asie (Chine, Inde, Japon) et d'Amérique latine (Argentine, Brésil, Chili, Mexique) ${ }^{76}$. Comme on l'a vu, les deux instruments Gautier destinés à l'observatoire de Rio de Janeiro furent une lunette photographique initialement destinée à la Carte du ciel ${ }^{77}$ et le grand cercle méridien dont nous avons retracé l'histoire. 


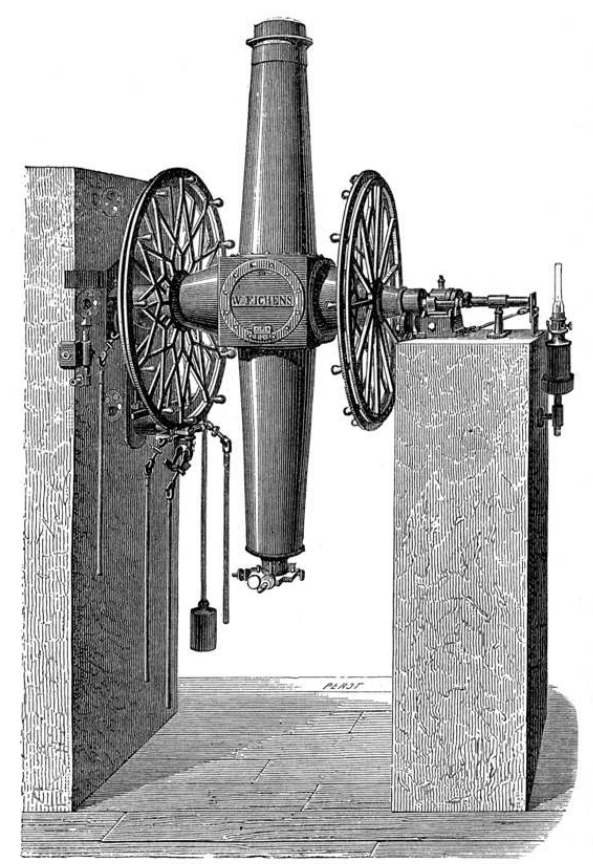

Fig. 7. - Cercle méridien de l'Observatoire de Lima.

Le cercle méridien Eichens de Lima (Pérou). ANDRÉ, Charles, ANGOT, André. L'astronomie pratique les observatoires en Europe et en Amérique, quatrième partie, Observatoires de l'Amérique du Sud. Paris : Gauthier-Villars, 1881, Figure 7.

Le destin de ce cercle méridien est original à plus d'un titre. Comme celui d'Eichens à Lima il resta en caisse à ses débuts ${ }^{78}$. Mais contrairement à celui-ci, au début du xxe siècle il devint un instrument opérationnel. Cependant, alors que les abris de tous les cercles méridiens français (Eichens, Gautier ou Brunner) installés peu après leur construction étaient des bâtiments en pierre à toiture à longs pans, les abris de ceux de Rio et de Tokyo ${ }^{79}$, installés au début du XXe siècle, furent en bois avec toit tonneau. Après des années de bons et loyaux services, pratiquement tous les cercles méridiens finirent par être considérés comme obsolètes. Dans le cas des instruments de construction française, seuls ceux de Bordeaux et de Tokyo poursuivirent leur carrière observationnelle grâce notamment à l'installation de caméras CCD. Dans certains des autres observatoires, ces instruments prestigieux restèrent sur place et relèvent désormais du patrimoine (Alger, Athènes ${ }^{80}$, Besançon, Hendaye, Lyon ${ }^{81}$, Paris, Toulouse) (fig. $\mathbf{n}^{\circ}$ 30). 
Figure 30

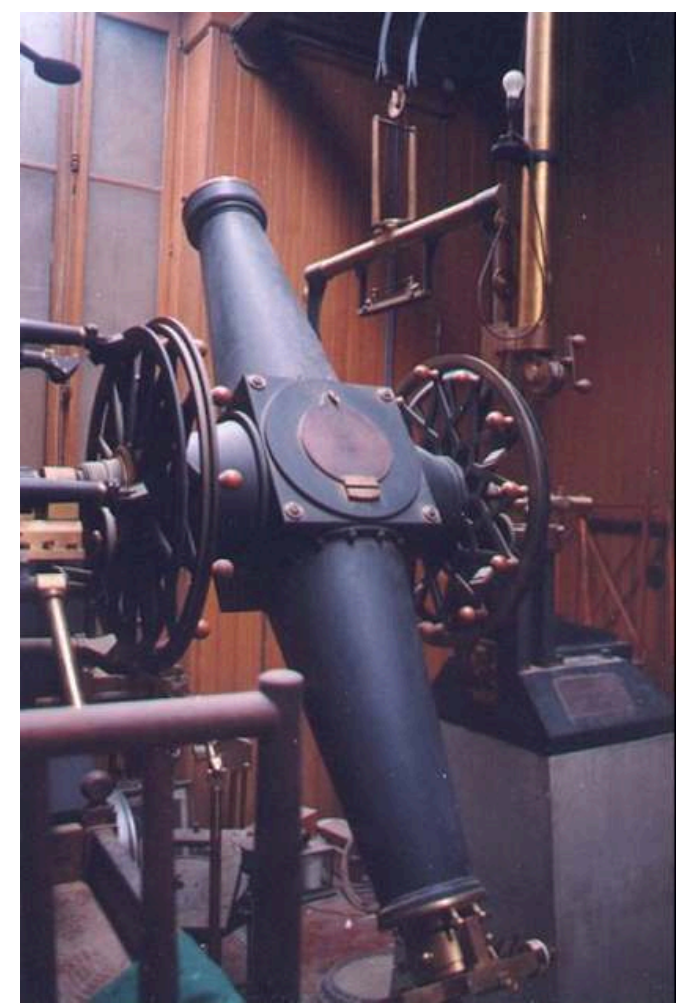

Le cercle méridien Gautier de l'observatoire d'Athènes (Grèce) en 2003.

(c) Archives Helléniques des Instruments scientifiques.

À Nice, l'instrument fut détruit à la fin des années 1960. Quant aux cercles méridiens de Marseille, La Plata et Rio, ils furent démontés, le premier en 1913 car le bâtiment fuyait, le second en $1938^{82}$, et celui de Rio en 1962 (fig. $\mathbf{n}^{\circ}$ 31). Là encore, le destin du cercle méridien de Rio se distingue des deux autres. Abandonné pendant plus de vingt ans, il intégra tout d'abord la riche collection fondatrice du MAST, celle des instruments obsolètes de l'observatoire national de Rio de Janeiro. Puis il fit l'objet d'un projet de restauration longuement réfléchi et élaboré. Enfin en 2003 - grâce au soutien de la fondation VITAE - l'opération de restauration de l'instrument débuta, opération couplée à celle de la construction in situ d'une réplique non fonctionnelle de l'abri destinée à la présentation au sein d'un espace muséal de l'instrument original ${ }^{83}$. 
Figure 31

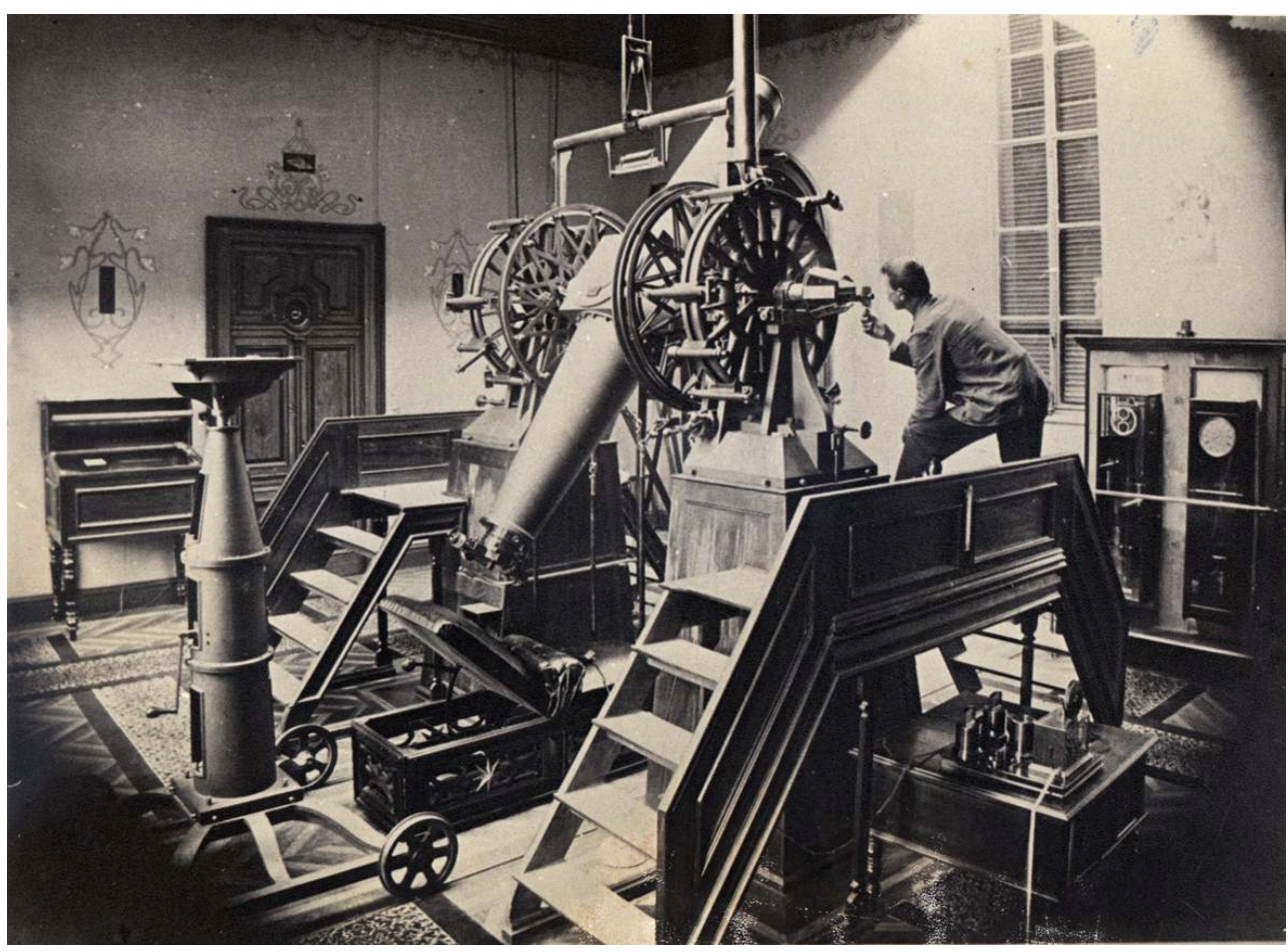

Le cercle méridien Gautier de l'observatoire de La Plata (Argentine) au début du XXe siècle.

Archives photographiques du Museo de Astronomia y Geofisica Observatorio astronomico de la Universidad nacional de La Plata, Argentine @ MAG/FCAG/UNLP.

Des quatorze cercles méridiens dus à des constructeurs français, celui de Rio de Janeiro est le seul à ce jour à être présenté au public à la fois in situ et dans un espace muséographique adaptés ${ }^{84}$. Il est également le seul à être intégré dans une collection d'instruments placée sous la responsabilité d'une équipe professionnelle. Conséquence du professionnalisme de ses travaux et preuve de la nécessité d'une telle institution, au cours des trois ou quatre années précédentes, le MAST a été sollicité par un nombre croissant d'institutions brésiliennes et latino-américaines désireuses de trouver appuis et conseils en matière d'opérations de conservation/restauration d'instruments scientifiques. La création du MAST a en effet provoqué la découverte de "collections » d'instruments sur tout le continent sud-américain, découverte ayant pour corollaire la nécessité de conserver ces objets d'intérêt historique et dans certains cas de les restaurer. Conscient de la nécessité de contribuer aux opérations de préservation de ces collections et en vue de répondre aux demandes de plus en plus nombreuses qui lui sont adressées concernant la formation et les prestations de services, le MAST est d'ailleurs sur le point d'implanter un nouveau laboratoire de conservation des instruments scientifiques dans un bâtiment annexe du musée. Une telle prise de conscience verra-t-elle le jour prochainement en France?

87 Les parties de cet article rédigées par Marcus Granato ont été traduites du portugais par Françoise Le Guet Tully. Les auteurs remercient Jean Davoigneau et Christine Etienne pour leur relecture attentive du manuscrit français, Paul Smith pour sa traduction du résumé et Marc Heller pour la reproduction des illustrations provenant des ouvrages de Liais. 


\section{BIBLIOGRAPHIE}

André, Charles, Angot, André. L'astronomie pratique : les observatoires en Europe et en Amérique, quatrième partie, Observatoires de l'Amérique du Sud. Paris : Gauthier-Villars, 1881, p. 29-48.

André, Louis. Scientific instrument restoration : Problems and practice at the Musée des Arts et Métiers and in France. The restoration of scientific instruments. Proceedings of the workshop held in Florence, December 14-15, 1998. Laura Manetti (éd.), Istituto e Museo di storia della scienza (éd.), Opificio delle pietre dure (éd.), Florence : Le Lettere, 2000, p. 23-30.

Ashley-Smith, Jonathan. The Ethics of Conservation. Care of Collections. Simon Knell (ed.), Leicester Readers in Museum Studies Series. London : Routledge, 1994, p. 11-20.

Astronomical register. Londres : J.D. Potter, vol. 23, n² 273, 1885, p. 217-223.

Auerback, Felix. The Zeiss Works and the Carl Zeiss Foundation in Jena : their scientific, technical and sociological development and importance popularly described. London : Foyle Ed., 1927, $273 \mathrm{p}$.

Barboza, Christina H. M. O encontro do Rei com Vênus. Thèse de master, Instituto de Ciências Humanas e Filosofia, Universidade Federal Fluminense, Niterói, Brésil, 1994.

Barreto, Luiz M. Observatório Nacional : 160 anos de história. Rio de Janeiro : Observatório Nacional, 1987.

Bonsanti, Giorgio. Once again on the restoration of scientific instruments. The restoration of scientific instruments. Proceedings of the workshop held in Florence, December 14-15, 1998. Laura Manetti (éd.), Istituto e Museo di storia della scienza (éd.), Opificio delle pietre dure (éd.), Florence : Le Lettere, 2000, p. 51-56.

Brenni, Paolo. $19^{\text {th }}$ Century French Scientific Instrument Makers : The Brunners and Paul Gautier. Bulletin of the Scientific Instrument Society, 1996, $n^{\circ} 49$, p. 38.

Brenni, Paolo. Better than new ? Scientific instrument restoration in Italy. The restoration of scientific instruments. Proceedings of the workshop held in Florence, December 14-15, 1998. Laura Manetti (éd.), Istituto e Museo di storia della scienza (éd.), Opificio delle pietre dure (éd.), Florence : Le Lettere, 2000, p. 89-97.

Brenni, Paolo. Instruments in South America : the collection of the Museu de Astronomia e Ciências Afins of Rio de Janeiro. Bulletin of the Scientific Instrument Society, 2000, nº 65, p. 25-28.

Briggs, John. A new firm of instruments makers. Astrophysical Journal. 1896, vol. 4, p 83.

Chinnici, Ileana. La Carte du ciel : genèse, développement et issues. La Carte du Ciel. Jérôme Lamy (éd.), Paris : EDP Sciences et Observatoire de Paris, 2008, p. 19-43.

Granato, Marcus, DUARTE, Jusselma, SUZUKI, Cristiane. Restauração do Pavilhão, Cúpula Metálica e Luneta Equatorial de $32 \mathrm{~cm}$ - Conjunto Arquitetônico do Museu de Astronomia e Ciências Afins (MAST). Anais do Museu Paulista. São Paulo ; Museu Paulista da USP, 2005a, v. 13, p. 273-314.

Granato, Marcus, SANTOS, Leandro Rosa dos, MIRANDA, Luiz Roberto M. de. Restauração de um teodolito astronômico da coleção do Museu de Astronomia e Ciências Afins - MAST (Brasil). Congresso latino-americano de restauração de metais, 2, 2005, Rio de Janeiro. Anais. Rio de Janeiro ; MAST, 2005b, p. 273-295. 
Icom, Committee for Conservation. Code of Ethics. [http://www.icom-cc.org.] [Date de consultation 07/02/2009]

Keene, Suzanne. Instruments of History : Appearance and evidence. The restoration of scientific instruments. Proceedings of the workshop held in Florence, December 14-15, 1998. Laura Manetti (éd.), Istituto e Museo di storia della scienza (éd.), Opificio delle pietre dure (éd.), Florence : Le Lettere, 2000, p. 57-68.

Launay, Françoise. Les acteurs de la mission de Jules Janssen au Japon en 1874. L'Astronomie, $n^{\circ} 121,2007$, p. 94-101.

Le Guet Tully, Françoise, Davoigneau, Jean. L'inventaire et le patrimoine de l'astronomie : l'exemple des cercles méridiens et de leurs abris. In Situ, $\mathrm{n}^{\circ} 6$, septembre 2005, 44 p. [http:// www.revue.inventaire.culture.gouv.fr/insitu/insitu/article.xsp ? numero $=6 \&$ id_article $=$ davoigneau-467\#] [date de consultation 06/02/2009].

Le Guet Tully, Françoise, DE LA NOË, Jérôme, SADSAOUD, Hamid. L'opération de la Carte du Ciel dans les contextes institutionnel et technique de l'astronomie française à la fin du XIX ${ }^{\mathrm{e}}$ siècle. La Carte du Ciel. Jérôme Lamy (éd.), Paris : EDP Sciences et Observatoire de Paris, 2008, p. 69-107.

Liais, Emmanuel. Annales de l'Observatoire impérial de Rio de Janeiro, Tome premier, Rio de Janeiro : Lombaerts \& Cie, 1882.

Liais, Emmanuel. L'espace céleste et la nature tropicale : Description physique de l'univers d'après des observations personnelles faites dans les deux hémisphères. Paris : Garnier frères, $1865,606 \mathrm{p}$.

Lourenço, Marta, Museu de Ciência da Universidade, Lisbonne. Communication personnelle (FLGT), 2008.

Mann, Peter R. Working exhibits and the destruction of evidence in the Science Museum. Care of collections, Simon Knell (ed.), Leicester Readers in Museum Studies Series. London : Routledge, 1994, p. 35-50.

Meehan, P. M. Is reversibility an option when conserving industrial collections ? Reversibility Does it exist? British Museum Occasional Paper, 135. Londres : British Museum, 1999, p. 11-15.

Miniati, Mara. Il Restauro degli Strumenti Scientifici. Il coltello di Delfo, Rome : Istituto Cultura Materiale e Archeologia Industriale, 1991, n 19, septembre, p. 13-18.

Miniati, Mara, BRENNI, Paolo. Restauro di strumenti storico-scientifici e filosofie di intervento. Restauro di strumenti i materialli : scienza, musica, etnografia. Bitelli, L. M. (ed.). Florence : Nardini Editore, 1993, p. 51-59.

Ministerio da Guerra. Relatório. Rio de Janeiro : Imprensa Nacional, 1891, p. 18-19.

Ministerio da Guerra. Relatório. Rio de Janeiro : Imprensa Nacional, 1894, p. 30-31.

Ministerio das industrias, VIAÇÃO E OBRAS PUBLICAS. Relatório. Rio de Janeiro : Imprensa Nacional, 1898, p. 15.

Ministerio das relações exteriores. Relatório. Rio de Janeiro : Imprensa Nacional, 1898.

Mohen, Jean-Pierre. Les sciences du patrimoine : identifier, conserver, restaurer. Paris : Odile Jacob, 1999, p. 181-232.

Morize, Henrique. Observatório Astronômico : um século de história (1827-1927). Rio de Janeiro : MAST/Salamandra, 1987. 
Mourão, Ronaldo Rogério de Freitas. The Brazilian contribution to the observation of the transit of Venus. Transits of Venus : New Views of the Solar System and Galaxy. Proceedings of the 196th Colloquium of the international astronomical union held in Preston, Lancashire, United Kingdom 7-11 June 2004, Kurtz, D.W. (ed.), Cambridge : Cambridge University Press, 2005, p. 154-160.

Muñoz-Viñas, Salvador. Contemporary theory of conservation. London : ButterworthHeinemann, 2005.

Museu de astronomia e ciências afins. Coordenação de Documentação em História da Ciência. Serviço de Arquivo de História da Ciência. Fundo : ON, Ofício nº 200, datado de 3 maio de 1913.

Museu de astronomia e ciências afins. Coordenação de Documentação em História da Ciência. Serviço de Arquivo de História da Ciência. Fundo : ON, Ofício datado de 7 de julho de 1915.

Museu de astronomia e ciências afins. Coordenação de Documentação em História da Ciência. Serviço de Arquivo de História da Ciência. Fundo : ON, Ofício n 177, datado de 30 de março de 1928.

Newey, Hazel. Conservation and the preservation of scientific and industrial collections. International congress of the International Institute for Conservation of Historic and Artistic Works (IIC), 18, 2000, Melbourne. Proceedings, Melbourne, Préprint. Communication personnelle (MG).

Pourbaix, Michel. Lectures on electrochemical corrosion. Bruxelles : Plenum Press, 1973.

Rolland-Villemot, Bénédicte. Une méthodologie pour la conservation et la restauration du patrimoine industriel, scientifique et technique. $13^{\text {th }}$ Triennal Meeting Rio de Janeiro 22-27 September - ICOM Committee for Conservation. London : James \& James Science, 2002, vol. 1, p. 187-191.

Sebastian, Andrés. Hacia una arqueología de la ciencia : problemas de conservación y restauración del patrimonio científico e industrial. Pátina, juin 1995, n 7, p. 118-129.

Stroobant, Paul. Les observatoires astronomiques et les astronomes. Bruxelles : Observatoire royal de Belgique, $1^{\text {ère }}$ édition 1907.

Stroobant, Paul. Les observatoires astronomiques et les astronomes. Bruxelles : Observatoire royal de Belgique, $2^{\mathrm{e}}$ édition 1937.

Syndicat des constructeurs en instruments d'optique \& de précision. L'Industrie française des instruments de précision, 1901-1902. Catalogue. Fac-similé, Paris : Alain Brieux, 1980, 271 p.

Tissandier, Gaston. Madame Emmanuel Liais. La Nature, Paris : G. Masson, 1874, deuxième semestre, p. 101-102.

Turner, Herbert Hall. The great star map, being a brief general account of the international project known as the Astrographic Chart. London : John Murray, 1912.

Videira, Antonio Augusto Passos. Os 175 anos do Observatório Nacional. Rio de Janeiro ; Observatório Nacional, 2002.

Videira, Antonio Augusto Passos. História do Observatório Nacional : Persistente construção de uma identitade cientifica. Rio de Janeiro : Observatório Nacional, 2007, 180 p.

Wheatley, Christopher J. Conservation and storage : scientific instruments. Manual of Curatorship. A guide to museum practice. London : Butterworth, 1986, p. 319-322.

Wolfschmidt, Gudrun, SEEMANN, A., KÜHL, D. Hamburger Sternwarte - Geschichte und Erhaltung, Hamburg : Förderverein Hamburger Sternwarte e. V., Band I, 2002. 


\section{NOTES}

1. ICOM, Committee for Conservation. Code of Ethics. [http://www.icom-cc.org.].

2. MINIATI, Mara. Il Restauro degli Strumenti Scientifici. Il coltello di Delfo, Rome: Istituto Cultura Materiale e Archeologia Industriale, 1991, n 19, septembre, p. 13-18.

3. SEBASTIAN, Andrés. Hacia una arqueología de la ciencia: problemas de conservación y restauración del patrimonio científico e industrial. Pátina, juin 1995, n 7, p. 118-129.

4. ANDRÉ, Louis. Scientific instrument restoration : Problems and practice at the Musée des Arts et Métiers and in France. The restoration of scientific instruments. Proceedings of the workshop held in Florence, December 14-15, 1998. Laura Manetti (éd.), Istituto e Museo di storia della scienza (éd.), Opificio delle pietre dure (éd.), Florence : Le Lettere, 2000, p. 23-30.

5. MEEHAN, P. M. Is reversibility an option when conserving industrial collections? Reversibility - Does it exist ? British Museum Occasional Paper, 135. Londres : British Museum, 1999, p. 11-15.

6. MANN, Peter R. Working exhibits and the destruction of evidence in the Science Museum. Care of collections, Simon Knell (ed.), Leicester Readers in Museum Studies Series. London: Routledge, 1994, p. 35-50.

7. NEWEY, Hazel. Conservation and the preservation of scientific and industrial collections. International congress of the International Institute for Conservation of Historic and Artistic Works (IIC), 18, 2000, Melbourne. Proceedings, Melbourne, Préprint. Communication personnelle (MG).

8. BRENNI, Paolo. Better than new ? Scientific instrument restoration in Italy. The restoration of scientific instruments. Proceedings of the workshop held in Florence, December 14-15, 1998. Laura Manetti (éd.), Istituto e Museo di storia della scienza (éd.), Opificio delle pietre dure (éd.), Florence : Le Lettere, 2000, p. 89-97.

9. MANN, P. R. Working exhibits and the destruction of evidence in the Science Museum. Care of collections, Simon Knell (ed.), Leicester Readers in Museum Studies Series. London : Routledge, 1994, p. 35-50.

10. Rolland-Villemot, Bénédicte. Une méthodologie pour la conservation et la restauration du patrimoine industriel, scientifique et technique. $13^{\text {th }}$ Triennal Meeting Rio de Janeiro 22-27 September - ICOM Committee for Conservation. London : James \& James Science, 2002, vol. 1, p. 187-191.

11. BONSANTI, Giorgio. Once again on the restoration of scientific instruments. The restoration of scientific instruments. Proceedings of the workshop held in Florence, December 14-15, 1998. Laura Manetti (éd.), Istituto e Museo di storia della scienza (éd.), Opificio delle pietre dure (éd.), Florence : Le Lettere, 2000, p. 51-56.

12. ASHLEY-SMITH, Jonathan. The Ethics of Conservation. Care of Collections. Simon Knell (ed.), Leicester Readers in Museum Studies Series. London : Routledge, 1994, p. 11-20.

13. IPHAN - Instituto do Patrimônio Histórico e Artístico Nacional.

14. BRENNI, Paolo. Instruments in South America : the collection of the Museu de Astronomia e Ciências Afins of Rio de Janeiro. Bulletin of the Scientific Instrument Society, 2000, $\mathrm{n}^{\circ} 65$, p. 25-28.

15. Liais fut le premier directeur astronome de l'observatoire de Rio de Janeiro, voir section 2.2

16. [date de consultation 06/02/2009].

17. Pour la plupart ces activités scientifiques relevaient d'initiatives de collèges jésuites. Quant aux académies scientifiques créées dans quelques villes brésiliennes durant la première moitié du XVII ${ }^{\mathrm{e}}$ siècle, leur durée de vie fut brève.

18. Alors sous influence britannique, la famille royale fuit le Portugal envahi par les armées napoléoniennes et se réfugia dans la capitale de sa vice-royauté brésilienne, Rio de Janeiro, laissant l'armée anglaise séjourner au Portugal. 
19. LOURENÇO, Marta, Museu de Ciência da Universidade, Lisbonne. Communication personnelle (FLGT), 2008.

20. MORIZE, Henrique. Observatório Astronômico : um século de história (1827-1927). Rio de Janeiro : MAST/Salamandra, 1987.

21. ANDRÉ, Charles, ANGOT, André. L'astronomie pratique : les observatoires en Europe et en Amérique, quatrième partie, observatoires de l'Amérique du Sud. Paris: Gauthier-Villars, 1881, p. 29-43 ; ASTRONOMICAL REGISTER. Londres : J.D. Potter, vol. 23, n² 273, 1885, p. 217-223.

22. LIAIS, Emmanuel. Annales de l'Observatoire impérial de Rio de Janeiro, Tome premier. Rio de Janeiro : Lombaerts \& Cie, 1882, p. 4. L'observatoire occupe un espace rectangulaire d'environ 2000 mètres carrés $(70 \mathrm{~m}$ x 27,30 m) orienté nord-sud. La terrasse sur laquelle sont installés les instruments se trouve à environ $60 \mathrm{~m}$ au-dessus du niveau de la mer.

23. VIDEIRA, Antonio Augusto Passos. Os 175 anos do Observatório Nacional. Rio de Janeiro: Observatório Nacional, 2002.

24. Selon André \& Angot (ANDRÉ, Charles, ANGOT, André. L'astronomie pratique : les observatoires en Europe et en Amérique, quatrième partie, Observatoires de l'Amérique du Sud. Paris : Gauthier-Villars, 1881, p. 31), l'observatoire possédait aussi un cercle mural et une lunette équatoriale de Dollond, mais ces instruments ne seront semble-t-il installés que plus tard, sous la direction de Liais.

25. Selon André \& Angot (ANDRÉ, Charles, ANGOT, André. L'astronomie pratique : les observatoires en Europe et en Amérique, quatrième partie, Observatoires de l'Amérique du Sud. Paris : Gauthier-Villars, 1881, p. 32), l'observatoire, déjà équipé pour la météorologie d'un baromètre de Fortin, d'un psychromètre d'August, d'un hygromètre de Regnault, fut doté en 1858 d'un anémomètre de Combes, d'un pluviomètre de Babinet, d'un actinomètre et d'un atmidoscope de Lerebours, d'un pyrhéliomètre de Pouillet et d'un «météorographe de Dollond, contenant baromètre, thermomètre, hygromètre, pluviomètre, évaporomètre, électromètre et anémomètre pour la vitesse et la force ".

26. ANDRÉ, Charles, ANGOT, André. L'astronomie pratique : les observatoires en Europe et en Amérique, quatrième partie, observatoires de l'Amérique du Sud. Paris: Gauthier-Villars, 1881, p. 30.

27. ANDRÉ, Charles, ANGOT, André. L'astronomie pratique : les observatoires en Europe et en Amérique, quatrième partie, Observatoires de l'Amérique du Sud. Paris : Gauthier-Villars, 1881, p. 30.

28. Remarqué par François Arago (1795-1853), Liais entra à l'observatoire de Paris en 1852. Deux ans plus tard, Leverrier (1811-1877), successeur d'Arago à la tête de l'Observatoire, le chargea de mettre en place un service météorologique international. En février 1854 il devint élèveastronome, en août de la même année astronome-adjoint et en 1857 astronome titulaire. À la suite de dissensions surgies en 1857 avec Leverrier, Liais demanda un congé de disponibilité. C'est alors qu'il décida de partir à ses frais au Brésil observer l'éclipse de soleil du 7 septembre 1858 .

29. LIAIS, Emmanuel. L'espace céleste et la nature tropicale : Description physique de l'univers d'après des observations personnelles faites dans les deux hémisphères. Paris : Garnier frères, 1865, p. 289. Liais disposa d'un observatoire à Olinda entre novembre 1859 et juin 1860. L'empereur s'y rendit deux fois en décembre 1859.

30. TISSANDIER, Gaston. Madame Emmanuel Liais. La Nature, Paris : G. Masson, 1874, deuxième semestre, p. 101-102.

31. Peu de temps après sa nomination à la tête de l'observatoire de Rio, Liais rentra en France. S'il commanda effectivement des instruments pour l'observatoire de Rio, il est probable qu'il prolongea son séjour en France en raison de l'état de santé de sa femme, atteinte de «fièvres intermittentes » contractées au Brésil en 1862. Celle-ci en mourut en juin 1874, Liais prit son poste à Rio au cours du second semestre de 1874 . 
32. MOURÃO, Ronaldo Rogério de Freitas. The Brazilian contribution to the observation of the transit of Venus. Transits of Venus : New Views of the Solar System and Galaxy. Proceedings of the 196th Colloquium of the international astronomical union held in Preston, Lancashire, United Kingdom 7-11 June 2004, Kurtz, D.W. (ed.), Cambridge : Cambridge University Press, 2005, p. 154-160 ; LAUNAY, Françoise. Les acteurs de la mission de Jules Janssen au Japon en 1874. L'Astronomie, $\mathrm{n}^{\circ}$ 121, 2007, p. 94-101.

33. BARBOZA, Christina H. M. O encontro do Rei com Vênus. Thèse de master, Instituto de Ciências Humanas e Filosofia, Universidade Federal Fluminense, Niterói, Brésil, 1994.

34. VIDEIRA, Antonio Augusto Passos. Os 175 anos do Observatório Nacional. Rio de Janeiro : Observatório Nacional, 2002, p. 25-26.

35. Cruls entreprit notamment de nombreuses expéditions scientifiques : à Punta Arenas (Chili), pour l'observation du passage de Vénus de 1882; sur le plateau central du Brésil, afin de déterminer le quadrilatère de Brasilia, site de la future capitale (1890); sur la frontière entre Pérou et Bolivie, afin de déterminer l'emplacement précis de la source de la rivière Javari, élément crucial dans le cadre du conflit frontalier entre les deux pays (MINISTERIO DAS RELAÇÕES EXTERIORES. Relatório. Rio de Janeiro : Imprensa Nacional, 1898).

36. L'opération de la Carte du ciel est un projet international ayant pour objectif la réalisation d'une carte photographique complète du ciel sur des plaques correspondant à environ $2^{\circ}$ de côté afin d'enregistrer toutes les étoiles jusqu'à la magnitude 14. Des plaques à plus courte durée de pose devaient par ailleurs permettre d'établir la position des étoiles jusqu'à la magnitude 11. Les travaux de la carte photographique et du catalogue devaient être effectués par dix-huit observatoires répartis dans les deux hémisphères : Greenwich, Rome, Catane, Helsinki, Potsdam, Oxford, Bordeaux, Toulouse, Alger, San Fernando, Tacubaya, Santiago, La Plata, Rio de Janeiro, Le Cap, Sidney et Melbourne (TURNER, Herbert Hall. The great star map, being a brief general account of the international project known as the Astrographic Chart. London: John Murray, 1912 ; CHINNICI, Ileana. La Carte du ciel : genèse, développement et issues. La Carte du Ciel. Jérôme Lamy (éd.), Paris : EDP Sciences et Observatoire de Paris, 2008, p. 25-31).

37. Actuellement dans le district de Santa Cruz, à l'ouest de la ville.

38. Pour diverses raisons, les cercles méridiens furent rapidement équipés de deux grands cercles gradués.

39. LE GUET TULLY, Françoise, DAVOIGNEAU, Jean. L'inventaire et le patrimoine de l'astronomie : l'exemple des cercles méridiens et de leurs abris. In Situ, $n^{\circ} 6$, septembre 2005, p. 7-19. [date de consultation 06/02/2009].

40. Georges Secrétan (1837-1906), neveu de Marc Secrétan (1804-1867), construisit notamment à la demande de Liais une lunette de 0,22 m pour l'observatoire de Rio de Janeiro. On lui doit aussi l'achèvement des télescopes de $0,83 \mathrm{~m}$ de l'observatoire de Toulouse et de 1,20 $\mathrm{m}$ de l'observatoire de Paris.

41. BRENNI, Paolo. $19^{\text {th }}$ Century French Scientific Instrument Makers: The Brunners and Paul Gautier. Bulletin of the Scientific Instrument Society, 1996, $n^{\circ} 49$, p. 38.

42. Peu après la proclamation de la République (15 novembre 1889), l'observatoire impérial de Rio de Janeiro changea de nom pour devenir le 31 mai 1890 l'observatoire de Rio de Janeiro (décret $n^{\circ} 451$ ) et passa sous la tutelle du ministère de la Guerre (MINISTERIO DA GUERRA. Relatório. Rio de Janeiro : Imprensa Nacional, 1891, p. 18-19).

43. MINISTERIO DA GUERRA. Relatório. Rio de Janeiro: Imprensa Nacional, 1891, p. 19. Cet instrument fut commandé en même temps que l'équatorial photographique pour l'opération de la Carte du ciel. Dans le même temps un nouveau site était recherché pour les installer (VIDEIRA, Antonio Augusto Passos. História do Observatório Nacional : Persistente construção de uma identitade cientifica. Rio de Janeiro : Observatório Nacional, 2007, p. 33). 
44. MINISTERIO DA GUERRA. Relatório. Rio de Janeiro : Imprensa Nacional, 1894, p. 31. D’après ce rapport, auraient été commandés en Europe l'équatorial photographique, le cercle méridien ainsi qu'un équatorial visuel.

45. MINISTERIO DAS INDUSTRIAS, VIAÇÃO E OBRAS PUBLICAS. Relatório. Rio de Janeiro: Imprensa Nacional, 1898, p. 125.

46. Détermination du quadrilatère destiné à la future capitale Brasilia sur le Planato du Brésil ; détermination des frontières avec la Bolivie et le Pérou (VIDEIRA, Antonio Augusto Passos. Os 175 anos do Observatório Nacional. Rio de Janeiro : Observatório Nacional, 2002, p. 34-35).

47. C'est également en raison de l'instabilité du terrain que le projet d'installation d'une grande lunette de $74 \mathrm{~cm}$ à l'observatoire de Paris dut être abandonné au début des années 1880. Dans ce cas l'instabilité résultait de la présence d'anciennes carrières dans le sous-sol (LE GUET TULLY, Françoise, DE LA NOË, Jérôme, SADSAOUD, Hamid. L'opération de la Carte du Ciel dans les contextes institutionnel et technique de l'astronomie française à la fin du XIX ${ }^{\mathrm{e}}$ siècle. La Carte du Ciel. Jérôme Lamy (éd.), Paris : EDP Sciences et Observatoire de Paris, 2008, p. 29).

48. MORIZE, Henrique. Observatório Astronômico : um século de história (1827-1927). Rio de Janeiro : MAST/Salamandra, 1987, p. 129.

49. MORIZE, Henrique. Observatório Astronômico : um século de história (1827-1927). Rio de Janeiro : MAST/Salamandra, 1987.

50. D'importants travaux de démolition étant alors entrepris dans le centre-ville et notamment dans le quartier du Morro do Castelo, à cette occasion coururent des rumeurs selon lesquelles des trésors cachés par les jésuites allaient être découverts...

51. BARRETO, Luiz M. Observatório Nacional : 160 anos de história. Rio de Janeiro: Observatório Nacional, 1987.

52. Ce qui explique la présence dans la collection du MAST de nombreux instruments météorologiques à côté des instruments astronomiques.

53. MUSEU DE ASTRONOMIA E CIÊNCIAS AFINS. Coordenação de Documentação em História da Ciência. Serviço de Arquivo de História da Ciência. Fundo : ON, Ofício $n^{\circ} 200$, datado de 03 maio de 1913.

54. Carl Zeiss (1816-1888) créa à Iéna en 1846 une entreprise d'optique et de mécanique de précision qui se spécialisa notamment dans la fabrication de microscopes (AUERBACK, Felix. The Zeiss Works and the Carl Zeiss Foundation in Jena: their scientific, technical and sociological development and importance popularly described. London : Foyle Ed., 1927, 273 p). Associé depuis 1866 avec Ernst Abbe (1840-1905), professeur à l'université de Iéna, Zeiss devint l'un des meilleurs fabricants d'optique du monde, notamment après qu'Abbe eut développé une théorie de la formation des images microscopiques qui permit l'élimination de l'aberration sphérique et de la coma dans la fabrication des lentilles. La société Zeiss est encore aujourd'hui l'un des leaders mondiaux dans le domaine de l'optique de haute technologie (http:// www.zeiss.fr/) [date de consultation 06/02/2009].

55. LE GUET TULLY, Françoise, DAVOIGNEAU, Jean. L'inventaire et le patrimoine de l'astronomie : l'exemple des cercles méridiens et de leurs abris. In Situ, $n^{\circ}$ 6, septembre 2005, p. 22-33.

56. WOLFSCHMIDT, Gudrun, SEEMANN, A., KÜHL, D. Hamburger Sternwarte - Geschichte und Erhaltung, Hamburg : Förderverein Hamburger Sternwarte e. V., Band I, 2002.

57. MUSEU DE ASTRONOMIA E CIÊNCIAS AFINS. Coordenação de Documentação em História da Ciência. Serviço de Arquivo de História da Ciência. Fundo : ON, Ofício datado, de 07 de julho de 1915.

58. MUSEU DE ASTRONOMIA E CIÊNCIAS AFINS. Coordenação de Documentação em História da Ciência. Serviço de Arquivo de História da Ciência. Fundo : ON, Ofício n 177, datado de 30 de março de 1928.

59. Créée en 1751 par Charles Leroy, horloger du roi, la maison Leroy inventa en 1757 le chronomètre de marine. Elle devint en 1808 l'un des fournisseurs officiels de la Marine nationale. 
En 1910 elle installa au sommet de la Tour Eiffel le premier système de transmission de l'heure par radio et devint un fabricant très réputé d'horloges astronomiques. [http://www.lleroy.com/] [date de consultation 06/02/2009].

60. William Gaertner (1865-1949) travailla chez les constructeurs d'instruments de précision Repsold à Hambourg, Breithaupt à Kassel et Hilger à Londres avant d'émigrer aux États-Unis où en 1896 il ouvrit à Chicago un petit atelier de construction d'instruments scientifiques sous le nom de Wm. Gaertner \& Co (BRIGGS, John. A new firm of instruments makers. Astrophysical Journal. 1896, vol. 4, p. 83). L'entreprise fabriqua notamment des instruments de précision pour l'observatoire Yerkes de l'université de Chicago. Inauguré en 1897, cet observatoire fut doté de la plus grande lunette astronomique du monde. Gaertner publia semble-t-il ses premiers catalogues d'instruments à partir de 1904. En 1923 l'entreprise s'installa dans de nouveaux locaux sous le nom de Gaertner Scientific Corporation. Son siège actuel se trouve toujours dans l'illinois. [http://www.gaertnerscientific.com/company_info.htm] [date de consultation 06/02/2009].

61. MORIZE, Henrique. Observatório Astronômico : um século de história (1827-1927). Rio de Janeiro : MAST/Salamandra, 1987.

62. Odílio Ferreira Brandão, qui travailla de nombreuses années comme volontaire aux côtés des personnels de la Coordination muséologique du MAST et contribua très efficacement à la collecte des instruments et à la constitution de la documentation les concernant.

63. IPHAN - Instituto do Patrimônio Histórico e Artístico Nacional

64. INEPAC - Instituto Estadual do Patrimônio Cultural

65. MINIATI, Mara, BRENNI, Paolo. Restauro di strumenti storico-scientifici e filosofie di intervento. Restauro di strumenti i materialli : scienza, musica, etnografia. Bitelli, L. M. (ed.). Florence : Nardini Editore, 1993, p. 55.

66. Les informations les plus précises provinrent des observatoires de Besançon et Toulouse.

67. On ignore pourquoi le micromètre Gautier d'origine ne fut pas utilisé lors de l'installation du cercle méridien. Selon le rapport envoyé au ministère de l'Agriculture (nouvelle tutelle de l'établissement) en 1923, un nouveau micromètre fut acquis chez le constructeur parisien Bouty. Ce micromètre fut fixé sur le socle du Gautier.

68. MINIATI, Mara, BRENNI, Paolo. Restauro di strumenti storico-scientifici e filosofie di intervento. Restauro di strumenti i materialli : scienza, musica, etnografia. Bitelli, L. M. (ed.). Florence : Nardini Editore, 1993, p. 53-54.

69. Muñoz-Viñas, Salvador. Contemporary theory of conservation. London: ButterworthHeinemann, 2005.

70. MOHEN, Jean-Pierre. Les sciences du patrimoine : identifier, conserver, restaurer. Paris : Odile Jacob, 1999, p. 181-232.

71. WHEATLEY, Christopher J. Conservation and storage : scientific instruments. Manual of Curatorship. A guide to museum practice. London : Butterworth, 1986, p. 319-322.

72. KEENE, Suzanne. Instruments of History: Appearance and evidence. The restoration of scientific instruments. Proceedings of the workshop held in Florence, December 14-15, 1998. Laura Manetti (éd.), Istituto e Museo di storia della scienza (éd.), Opificio delle pietre dure (éd.), Florence : Le Lettere, 2000, p. 57-68.

73. GRANATO, Marcus, DUARTE, Jusselma, SUZUKI, Cristiane. Restauração do Pavilhão, Cúpula Metálica e Luneta Equatorial de $32 \mathrm{~cm}$ - Conjunto Arquitetônico do Museu de Astronomia e Ciências Afins (MAST). Anais do Museu Paulista. São Paulo ; Museu Paulista da USP, 2005a, v. 13, p. 273-314; GRANATO, Marcus, SANTOS, Leandro Rosa dos, MIRANDA, Luiz Roberto M. de. Restauração de um teodolito astronômico da coleção do Museu de Astronomia e Ciências Afins MAST (Brasil). Congresso latino-americano de restauração de metais, 2, 2005, Rio de Janeiro. Anais. Rio de Janeiro ; MAST, 2005b, p. 273-295. 
74. Dans ce type de corrosion, le métal est piqué de façon très localisée par les agents corrosifs, ce qui provoque de petites perforations qui ressemblent à des trous d'épingle (POURBAIX, Michel. Lectures on electrochemical corrosion. Bruxelles : Plenum Press, 1973).

75. Les Brunner ont notamment construit le grand cercle méridien de l'observatoire de Nice ainsi qu'une lunette équatoriale à l'observatoire de Lyon et une à l'observatoire de Toulouse.

76. Voir extrait du Catalogue de Gautier 1901-1902 [figures 13 et 14]. L'étude du développement de cette sphère d'influence comparée à celle des grands constructeurs allemands et anglais reste à faire.

77. L'opération de la Carte du ciel est un projet international ayant pour objectif la réalisation d'une carte photographique complète du ciel sur des plaques correspondant à environ $2^{\circ}$ de côté afin d'enregistrer toutes les étoiles jusqu'à la magnitude 14. Des plaques à plus courte durée de pose devaient par ailleurs permettre d'établir la position des étoiles jusqu'à la magnitude 11. Les travaux de la carte photographique et du catalogue devaient être effectués par dix-huit observatoires répartis dans les deux hémisphères : Greenwich, Rome, Catane, Helsinki, Potsdam, Oxford, Bordeaux, Toulouse, Alger, San Fernando, Tacubaya, Santiago, La Plata, Rio de Janeiro, Le Cap, Sidney et Melbourne (TURNER, Herbert Hall. The great star map, being a brief general account of the international project known as the Astrographic Chart. London: John Murray, 1912 ; CHINNICI, Ileana. La Carte du ciel : genèse, développement et issues. La Carte du Ciel. Jérôme Lamy (éd.), Paris : EDP Sciences et Observatoire de Paris, 2008, p. 25-31).

78. En ce qui concerne l'observatoire de Lima, André \& Angot (ANDRÉ, Charles, ANGOT, André. L'astronomie pratique : les observatoires en Europe et en Amérique, quatrième partie, Observatoires de l'Amérique du Sud. Paris: Gauthier-Villars, 1881, p. 44-48) indiquent que «[...] les révolutions [...] ont empêché de donner suite au projet primitif. Les instruments sont encore actuellement dans leurs caisses, et rien ne fait espérer qu'on entreprenne prochainement la construction de l'Observatoire lui-même. "

79. Le cercle méridien de Tokyo figure dans le catalogue 1901-1902 de Gautier, il n'est pas mentionné dans la première édition de l'ouvrage de Stroobant sur les observatoires (STROOBANT, Paul. Les observatoires astronomiques et les astronomes. Bruxelles: Observatoire royal de Belgique, 1907), mais est mentionné dans la deuxième édition du même ouvrage en 1937. Comme il semble qu'il n'a pas été mis en service sur le site sur lequel fut implanté l'observatoire de Tokyo en 1888, mais sur le site où il fut transféré en 1924 (situé à Mitaka à une vingtaine de kilomètres de Tokyo), il se peut qu'il ait été commandé à Gautier vers 1900 mais mis en service seulement en 1924.

80. Pour le cercle méridien Gautier de l'observatoire d'Athènes voir le site des Archives Helléniques des Instruments scientifiques.[date de consultation : 25/03/2009].

81. Le cercle méridien de l'observatoire de Lyon sera prochainement exposé non pas in situ à l'observatoire, mais au musée des Confluences [date de consultation : 06/02/2009].

82. Le cercle méridien de La Plata fut commandé à Gautier en 1887, terminé en 1889 et exposé lors de l'Exposition universelle de Paris. Il est livré à l'observatoire en 1890, il ne fut installé qu'en 1903 et démonté dès 1938 pour être remplacé sur les mêmes piliers et dans le même abri par un cercle méridien commandé en 1906 au constructeur allemand Repsold et livré en Argentine en 1908. Actuellement, le cercle Gautier est toujours conservé démonté dans les soussols de l'observatoire. Observatorio astronomico de la Universidad nacional de La Plata. Publicaciones, Tomo I. Descripción general del Observatorio, su posición geográfica y observaciones de cometas y estrellas dobles. La Plata, 1914 et Circulo Meridiano Gautier, Museo de Astronomico y Geofisica, Observatorio Astronomico de la Universidad Nacional de La Plata. Informations transmises par Sofia A. CORA, Observatorio Astronómico, Universidad Nacional de La Plata, Argentina, mars 2009. 
83. Une opération quelque peu similaire est entreprise à l'observatoire de Marseille. Le cercle méridien Eichens démonté en 1913 est en cours de restauration mais il n'est cependant pas envisagé de reconstruire le bâtiment démoli il y a près d'un siècle.

84. À l'observatoire d'Abbadia (Hendaye), le cercle méridien est présenté au public dans le cadre d'une visite générale du château.

\section{RÉSUMÉS}

Après l'énoncé de quelques principes concernant la restauration d'instruments scientifiques et la présentation de la politique mise en place au musée d'astronomie du Brésil (MAST), nous dressons un bref historique de l'observatoire national de Rio de Janeiro et présentons l'histoire de l'un de ses grands instruments, un cercle méridien commandé à la fin du XIXe siècle au célèbre constructeur français d'instruments de précision Paul Gautier. Nous relatons ensuite comment ce cercle méridien - démonté dans les années 1960 - a fait l'objet d'une importante opération de restauration, opération dont l'aboutissement fut l'inauguration en 2006 du nouvel espace muséal qui abrite désormais l'instrument.

This article opens with some general remarks on the principles of restoring scientific instruments and on the presentation of the restoration policies of the Brazilian museum of astronomy (MAST). It then gives a brief history of the astronomical observatory at Rio de Janeiro and presents in particular the history of one of its major instruments, a meridian circle built for the observatory at the end of the 19th century by the celebrated French maker of scientific instruments Paul Gautier. It describes how this meridian circle, dismantled during the 1960 s and given to the museum of astronomy (MAST) has recently been the object of an important restoration programme. This operation led to the inauguration, in 2006, of a new space in the museum devoted to this instrument.

\section{INDEX}

Keywords : Astronomical Observatory, Bergedorf Observatory, Carte du Ciel (Sky Chart), Meridian Circle, Paris Observatory, Restoration, Rio de Janeiro Observatory, Scientific and Technical Museum, Scientific Instruments

Mots-clés : Carte du ciel, cercle méridien, Emmanuel Liais, éthique, Henrique Morize, ICOM, instruments scientifiques, John Dollond, Louis Cruls, MAST, musée des sciences et des techniques, observatoire astronomique, observatoire de Bergedorf, observatoire de Paris, observatoire de Rio de Janeiro, Paul Gautier, Repsold, restauration, Science Museum, Zeiss

\section{AUTEURS}

\section{MARCUS GRANATO}

Museu de Astronomia e Ciências Afins, Rio de Janeiro, Brésil. marcus@mast.br 


\section{FRANÇOISE LE GUET TULLY}

University of Nice Sophia Antipolis, CNRS, Observatoire de la Côte d'Azur. leguet@oca.eu 